\title{
Using Images to Help Children Talk about Their
}

\section{Experiences}

\author{
A Thesis Submitted to \\ Victoria University of Wellington \\ In Fulfilment of the Requirements for the Degree of \\ Master of Science in Psychology
}

by

Wendy Lilian Higgs

April 2013 


\begin{abstract}
Prior research evaluating the effect of Human Figure Diagrams (HFDs) on the nature of children's recall of touch related information has yield mixed findings. We examined the nature of information elicited by 9-11 year old children $(n=55)$ following participation in a scripted event that incorporated instances of physical contact/touch. We examined the amount and accuracy of information reported under the following conditions; with the aid of a HFD, a photograph of the child, or verbal prompts only. Children presented with HDFs or photographs reported significantly more information following an exhaustive verbal interview, than children provided with verbal prompts only. The accuracy of reports was high overall and did not differ significantly by condition. When children were asked focussed questions about instances of both true and false touch that was not reported during the verbal interview, the accuracy of their accounts reduced significantly compared to phases of the interview that were predominantly child-led. This suggests that visual aids may be safe to use to clarify/elaborate on already reported information, at least with this age group. Specific questions about instances of unreported touch should be avoided.
\end{abstract}




\section{Acknowledgements}

I would firstly like to thank my supervisor Dr. Deirdre Brown for her guidance and support throughout this process, for always being positive, and for making time for me beyond what I could have expected.

Thank you to all of my incredible family and friends for their support and patience throughout this year. Thank you especially to my parents for their continuing support throughout my education.

I am grateful to all of the research assistants who were involved in data collection and data processing, and to everybody in the laboratory who offered their guidance and support during this study.

This research would not have been possible without the Wellington schools who agreed to participate in our study, and all of the children and their parents who consented to and were involved in this research. 


\section{Table of Contents}

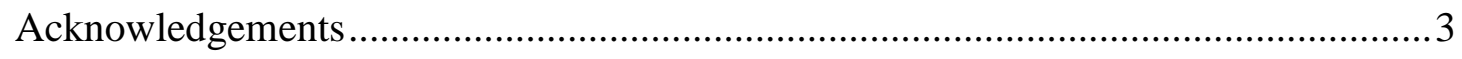

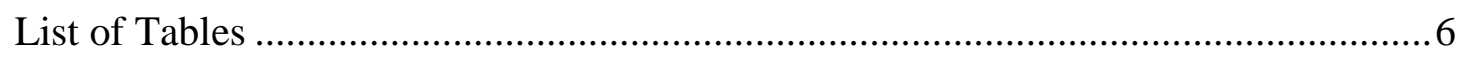

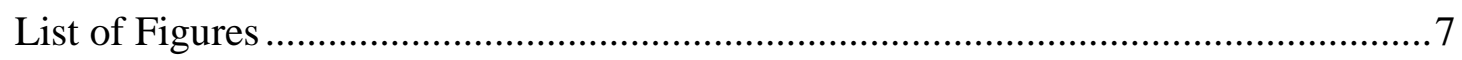

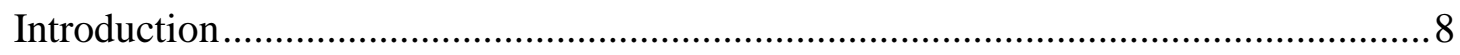

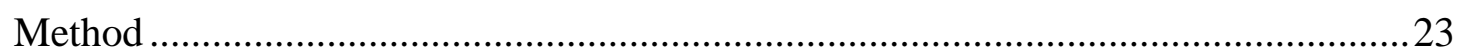

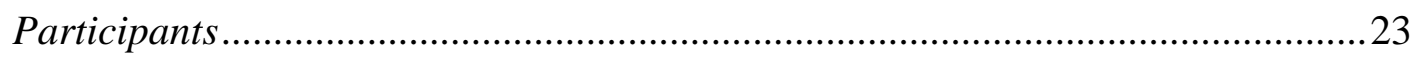

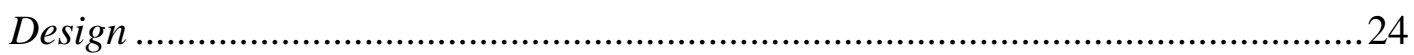

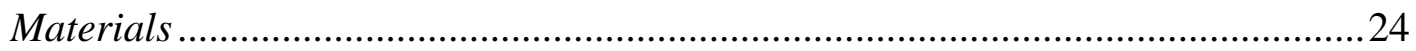

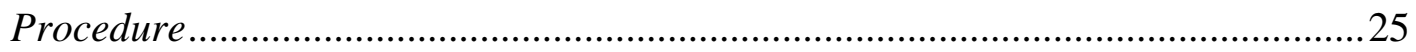

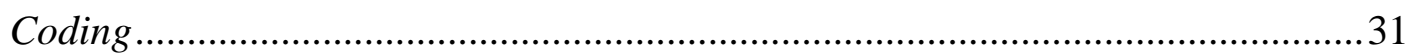

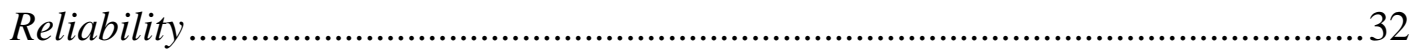

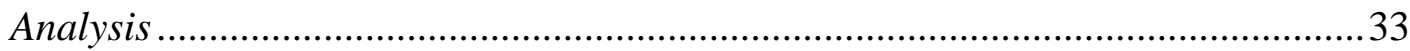

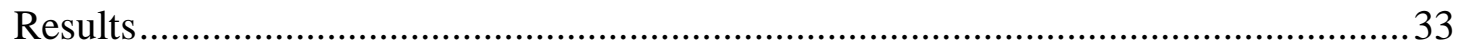

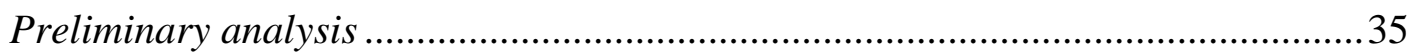

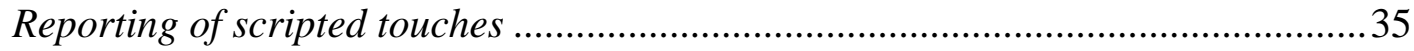

Spontaneous recall and reporting of touch-related information .............................36

Reporting of touch-related information following introduction of visual aids.........36

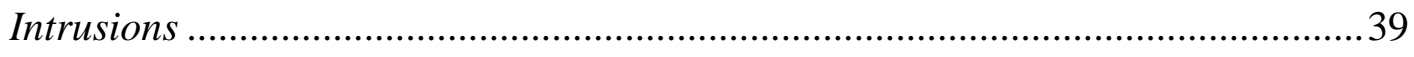

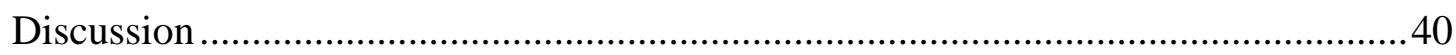


Reporting of scripted touches 40

Spontaneous recall and reporting of touch-related information....

The relationship between amount and accuracy of information reported. 42

Reporting of touch-related information following introduction of visual aids ........42

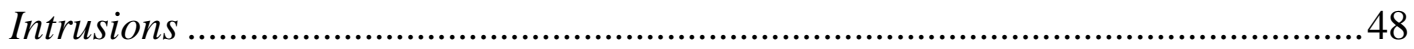

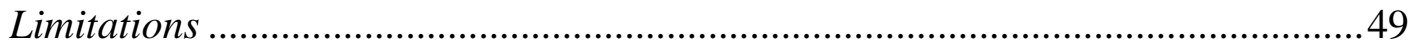

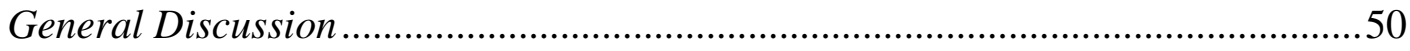

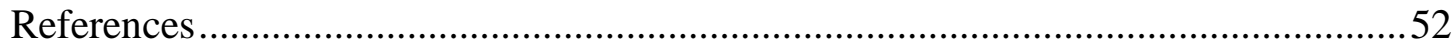

APPENDIX A: Parent information letter ………...............................................57

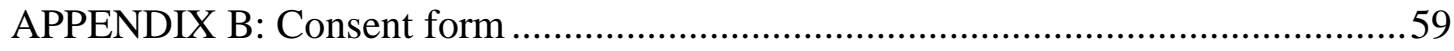

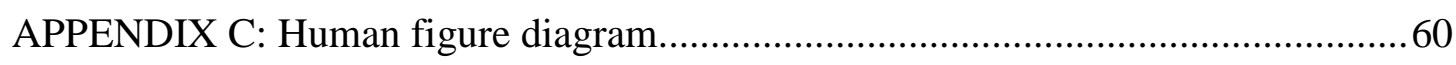

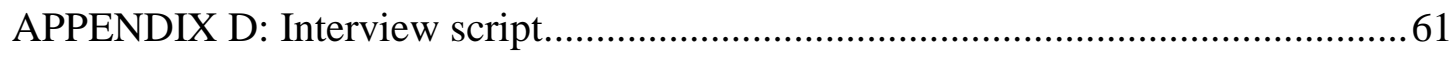

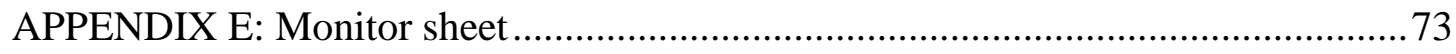

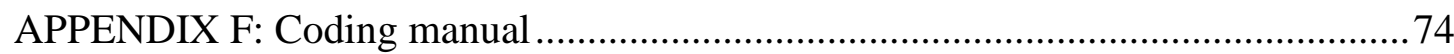




\section{List of Tables}

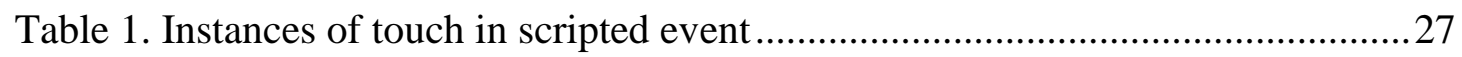

Table 2. Mean (SD) number of details and accuracy, and proportion of scripted

touches reported by children during the verbal interview. 36

Table 3. Mean $(S D)$ number of details and accuracy of touch information reported during phases 2 and 3 of the interview. .37 


\section{List of Figures}

Figure 1. Amount of information elicited in touch follow up (phase 2) and specific touch questions (phase 3) phases of the interview by condition...... 38

Figure 2. Accuracy of information elicited in touch follow up (phase 2) and specific touch questions (phase 3) phases of the interview by condition. 


\section{Using images to help children talk about their experiences}

Child maltreatment is particularly topical in New Zealand, with a number of recent cases being subject to extensive media attention. Child sexual abuse is associated with a number of established adverse short term and long term psychological, physical and social outcomes. Child maltreatment can disrupt early brain development, with extreme stress potentially impairing development of nervous and immune systems (WHO, 2010). Established long term outcomes of child sexual abuse include increased risk for developing psychiatric difficulties, such as eating disorders, depression and substance use problems, as well as earlier consensual sexual activity, early unintended pregnancy, risky sexual behaviours, sexually transmitted infections, and exposure to subsequent abuse (Fanslow, Robinson, Crengle, \& Perese, 2007). The World Health Organisation also identify economic impact related to costs of hospitalisation, mental health treatment, child welfare, and management of chronic health conditions following a disproportionately high prevalence of smoking and obesity (2010).

It is difficult to determine the precise prevalence of child sexual abuse rates in New Zealand due to the range of different methodologies and definitions employed between existing studies. However it is clear that it is a significant issue in this country, with existing studies stating that up to one in three New Zealand women report having experienced some form of child sexual abuse. For example, Anderson, Martin, Mullen, Romans and Herbison (1993) report that 32\% of a sample of 2000 woman participating in the Otago Women's Health study had experienced some form of sexual abuse prior to age 16 . Furthermore, they report that $16 \%$ of these incidents had occurred when the women were younger than 12 years of age, with 8 to 12 year old children identified as most at risk (Anderson, et al., 1993). A second study based 
on a sample of 2,855 women aged 18-64 from Auckland and Waikato regions stated that $23.5 \%$ of women from the Auckland region and $28.2 \%$ of women from the Waikato region had reported some form of sexual abuse prior to age 15 , with the median age of the first incident being at 9 years (Fanslow, Robinson, Crengle, \& Perese, 2007). Finally, The New Zealand Youth 2000 study surveying almost 10,000 secondary students reported that $26 \%$ of females and $14 \%$ of males disclosed that they had experienced some form of sexual abuse (Fleming, Merry, Robinson, Denny \& Watson, 2007).

The issue of non-disclosure is intrinsic to the nature of child sexual abuse and also impacts upon prevalence data. In the study by Anderson et al., (1993) described above, authors found that only $7.5 \%$ of child sexual abuse cases were ever officially reported. Wilson (2007) comments on data collected in New Zealand from evidential interviews for sexual abuse conducted between 2000 and 2002. In this data set, 4060 children were interviewed for concerns about sexual abuse and of these, 1316 made no allegation throughout the duration of the interview process (Wilson, 2007). While there is no means of determining the proportion conclusively, it is likely that this figure incorporates at least some instances of false negative reporting, particularly given that $24 \%$ of these cases were assessed as having a continued level of concern, and a further $24 \%$ were considered too young to complete the interview process (Wilson, 2007).

Historically children's eyewitness accounts have been considered with caution due to questions about children's capacity to accurately report events (Ceci \& Bruck, 1993). The assumption that children are highly suggestible has portrayed them as unable to give reliable testimony (Malloy \& Quas, 2009). This is problematic given that child eyewitness testimony is often relied upon heavily, if not exclusively, as 
evidential in child sexual abuse cases (Malloy \& Quas, 2009). As such, a great deal of research has been conducted around verbal interviewing techniques with children, and this has highlighted a number of potential areas of influence in children's ability to make accurate and complete reports about things that have happened to them.

There are a number of cognitive competencies that are still undergoing development in children who are required to give eye witness testimony (see Pipe, Lamb, Orbach \& Esplin, 2004 for a review). Developmental competencies associated with age are established to influence the accuracy and detail of children's reports (Pipe et al., 2004). For example compared with adults, children are still developing their semantic knowledge about world events, their ability to use schemas or scripts effectively, and their memory strategies for the encoding, storing and retrieval of information (Klemfuss \& Ceci, 2009). They also have a limited attentional capacity and reduced processing speed relative to that of an adult (Klemfuss \& Ceci, 2009). As such, children's reports of things that have happened to them are generally very brief, and this is thought to be due to their more limited competencies in language use, knowledge of world events, memory capacity, and reporting strategies (Pipe et al., 2004).

These developing competencies become further strained by typical forensic interviewing processes where there is likely to be a long delay between the child being exposed to an incident of abuse and then being asked to recall and report on that experience. As the passage of time between the event and the recall increases, so does that number of errors in recall (Pipe et al., 2004). However despite historical assumptions about the accuracy of child eye witness testimony, even very young children are able to provide largely accurate reports of their experiences (e.g. Marin, Holmes, Guth \& Kovac, 1979; Lamb et al., 2003). Furthermore, when children do 
make errors in reporting, they are more likely to make errors of omission than errors of commission, that is, they are more likely to omit information completely than they are to report something that is entirely false (e.g. Goodman \& Reed, 1986). Errors of omission become of particular concern during evidential interviewing with children, given the brevity of their reports to begin with (Pipe et al., 2004).

The quality of the interaction between the child and the interviewer and the retrieval mechanisms used to elicit reports of children's experiences is also established to influence the amount and the accuracy of the information reported (Pipe et al., 2004). Given the nature of children's brief accounts of events in the first place, the use of interviewing techniques that elicit more detailed information without eliciting false information are essential. Different memory processes are engaged by using different types of interviewing techniques. For example free recall techniques in interviewing, such as "tell me everything you remember about ..." are demonstrated to elicit brief, but most likely accurate accounts of events (Pipe et al., 2004). Children are often able to recall additional details in response to open-ended recall questions such as "tell me more about that" or recall prompts such as "when did that happen?" In comparison, recognition techniques, including yes/no questions and forced-choice prompts such as "did he have glasses?" or "did this happen in the day or at night?" increase the probability of eliciting erroneous reports of events (Lamb, Orbach, Hershkowitz, Horowitz \& Abbott, 2007). As such, investigative interviewers have been encouraged to prioritise interview techniques that engage recall memory processes, and to minimise interview techniques that engage recognition memory processes.

In light of consensus from child eyewitness testimony and forensic interviewing strategy research, professional bodies have developed evidence based 
guidelines for conducting developmentally appropriate investigative interviews with children. However, studies have since indicated that despite intensive training in best practice investigative interviewing, forensic interviews were still not being conducted accordingly (e.g. Aldridge \& Cameron, 1999). Following these findings, Orbach et al. (2000) developed the National Institute of Child Health and Human Development (NICHD) Investigative Interview Protocol from research in the area, to guide forensic interviewers in conducting developmentally appropriate interviews with children. Interviewing processes currently adopted in New Zealand are based on extensive research of developmentally appropriate verbal interviews with children, and are outlined in Wilson (2007).

Lamb, Orbach, Hershkowitz, Esplin and Horowitz (2007) reviewed studies designed to evaluate the effect of the NICHD protocol on the quality of investigative interviews. They reported that the protocol improved reliability dramatically dissimilarly to any other technique investigated (Lamb et al., 2007). In 2009 Lamb et al. examined children's reports elicited from forensic interviews, when following the NICHD Structured Interview Protocol compared with alternative guidelines. They found that compared with alternative guidelines, children interviewed under the NICHD protocol reported more information in response to free-recall prompts and less information in response to more focussed interviewer-led questions. This means that interviewers were utilising more prompts demonstrated to elicit largely accurate information, and less focussed questions demonstrated to elicit increased instances of false reporting. This demonstrates that when given a specific structured interview protocol to follow, interviewers are more likely to adhere to best practise guidelines. Current New Zealand investigative interviewing guidelines are adapted from this protocol, and it has been adopted in many other countries as best practice. 
Alongside verbal techniques, forensic interviewers frequently use non-verbal strategies. These techniques may be employed to clarify or elicit further information following ambiguous verbal reports of touch instances. Such detail is often necessary to establish the nature of the charges to be laid in a child sexual abuse case.

Furthermore, given the lack of corroborating evidence typically available in child sexual abuse cases, eliciting more detailed accounts from children is important in order to progress with investigations. It follows that any potential method of clarifying and/or eliciting additional touch related detail should be investigated to establish its efficacy and its safety for use in forensic contexts.

Initially there was widespread use of anatomical dolls in forensic interviewing. This was following the rationale that the use of dolls may help to address potential language, socio-motivational and cognitive issues associated with verbal communication of touch related information with children. However despite their intuitive appeal, evidence has since discouraged use of dolls in forensic interviewing following findings that firstly, dolls were not helpful in eliciting additional information, and secondly that they elicited increased errors in reporting (Salmon, 2001). One problematic aspect of using dolls as an interviewing aid is that they hold an association with fantasy play. The task of differentiating between times when a doll is a plaything, and times when a doll is to be used as a symbolic representation of themselves is thought to be taxing upon children, given their still developing cognitive competencies in this area (DeLoache \& Marzolf, 1995) Secondly, using dolls requires children to have the developmental capacity to recognise and use them as symbolic representations of themselves, while simultaneously engaging in the cognitive task of recalling and reporting their experiences. Given children's still developing competencies in attention, processing speed, symbolic representation and 
memory capacity (Klemfuss \& Ceci, 2009) it is likely that the strain of multitasking impacts upon their ability to accurately recall and report their experiences.

Human Figure Diagrams (HFDs) are two dimensional representations of the human body (Pipe \& Salmon, 2009). HFDs are currently being investigated as a potentially safer alternative to anatomical dolls. HFDs may, like dolls, also provide children with an opportunity to respond non-verbally to touch related enquiry and may address the language, socio-motivational, and cognitive difficulties identified with verbal only interviewing. Firstly, they may assist children in communicating their experiences, particularly with very young children given the limitations of their still developing language abilities (Pipe \& Salmon, 2009). Secondly, they may provide children with a non-verbal means of communicating potentially shameful or embarrassing information, particularly in older children who are developing a greater awareness of the nature of social evaluation (Pipe \& Salmon, 2009). Thirdly, they may serve as an external memory cue for children regardless of their age, when verbal accounts of their experiences are incomplete or unclear (Pipe \& Salmon, 2009).

Children's limited ability to use dolls as aids to communicate information has been attributed largely to their limited skills in understanding and using symbolic representations. HFDs may reduce the impact of this in two ways. Firstly, DeLoache and Marzolf (1992) specify that the symbolic nature of pictures is more easily appreciated by children that that of dolls. Part of this may be because they eliminate or at least reduce the dual association with fantasy play that dolls appear to encourage (Pipe \& Salmon, 2009). Secondly, given that HFDs may provide a more easily accessible symbolic representation, it is also possible that they could reduce the cognitive load upon children of having to process symbolic a representation while 
also recalling and reporting information, and therefore serve as a more effective external memory cue.

HFDs may be used in a number of ways during investigative interviews. For example, they can be used to establish names for body parts, to elicit information regarding touch, or to clarify already reported verbal touch information (Pipe \& Salmon, 2009). The timing of the introduction of HFDs can also vary, alongside their purpose. HFDs have been introduced at the beginning of the interview to determine children's definitions of body parts, during the interview when a child reports an incidence of touch to clarify the information, at the end of the interview to clarify already reported information about touch, and at the end of the interview to elicit any further information. In New Zealand, protocol around non-verbal interviewing techniques allows for use of non-anatomically detailed body diagrams to clarify body parts, and clothed dolls to clarify body positioning following ambiguous verbal reports (Wilson, 2007). Currently there is no consensus as to how HFDs should be used in forensic interviewing, if at all.

When HFDs are used to for the purpose of clarifying or elaborating upon already reported information they are used in the context of free recall prompting. We know from literature examining verbal interviewing techniques that recall prompts are likely to elicit largely accurate information (Lamb et al., 2007). In comparison when HFDs are used for the purpose of eliciting new information they are likely to be used in the context of recognition prompting. We know from literature that recognition prompts are likely to elicit a decreased amount of information relative to recall prompts and are more likely to elicit inaccurate information, as children may not have encoded the information they are being asked about in the first place (Lamb et al., 2007). The present study seeks to compare the amount and accuracy of touch related 
information elicited using a HFD to clarify already reported touch information, compared with using a visual aid to elicit new touch information. We seek to do this by introducing visual aids, following an extensive verbal interview, as a second opportunity to recall and clarify any touch related information children have previously reported. We then propose to ask about both true and false instances of unreported touch with reference to the visual aid.

HFDs require an element of cognitive competency in symbolic representation given that they may differ in resemblance to the child's own body composition, gender, ethnicity and age, and remain unrealistic at least to a certain degree. A photograph of the child may reduce the cognitive load that this imposes. With the advent of digital photography and portable printing devices, a photograph potentially offers a readily accessible visual aid to support children's recall and reporting. This may lead to increased accuracy of reported touch information. Furthermore, according to encoding principles, actual items from an experienced event should make the most effective retrieval cues (Pipe \& Salmon, 2009). As such photographs may provide an even stronger retrieval cue than HFDs given that they represent the child themselves. This may lead to an increase in the quantity of touch information children are able to report. In addition, photographs may serve to decrease ambiguity about the specific location of instances of touch reported during interviewing. HFDs currently vary in the degree to which they mirror the body composition of the child, particularly in regard to cartoon like or 'gingerbread' HFDs. When a child specifies a touch on a photograph of themselves as opposed to on an HFD, it is less likely that the location of the touch will be ambiguous and thus require further clarification. It may be that this leads to more accurate reporting of touch information. 
There are a small number of studies overall investigating the effect of introducing HFDs during the interview process on the amount and accuracy of information elicited. Studies have demonstrated mixed support for HFD use, and present a number of methodological variances and limitations. Two studies demonstrate support for use of HDFs in eliciting additional information following verbal interviewing. In a field study Aldridge et al., (2004) examined interviews conducted following the NICHD protocol by police officers with children who were alleged victims of sexual abuse. They found that children aged 4-13 who were presented with an HFD elicited 86 new forensically relevant details post the verbal interview (Aldridge et al., 2004). HFDs appeared most useful in children aged 4-7 years; recall increased by $27 \%$ following introduction of the visual aid.

Teoh, Yang, Lamb and Larsson (2010) coded transcripts from Aldridge et al.'s study (2004) to examine the nature of the additional information that was elicited following the introduction of a HFD. They looked at type of information according to whether it was a repeated, or previously unreported touch, and the clarity of the touch reports. They found that HFDs elicited both new reports of touch instances, and elaborations on already reported touch. Given the less developmentally appropriate nature of the questions that elicited the new information, concerns were raised about its accuracy, and due to the nature of field research could not be established. With respect to clarification of already reported touch information, Teoh et al., (2010) found that HFDs were most beneficial for older as opposed to younger children.

Several studies examining HFD use have demonstrated that they appear to elicit false reports of touch, including forensically relevant touch. Steward et al., (1996) interviewed children aged 3-6 years post undergoing a medical procedure involving genital contact. They found that children interviewed with HFDs reported 
more information than children interviewed without HFDs, but that this was at the expense of accuracy. Specifically, children interviewed with HFDs reported more false incidents of touch to the genital and anal areas than children interviewed without HFDs. In a laboratory based study, Willcock, Morgan and Hayne (2006) interviewed children aged 5-6 years with a HFD either immediately after a target event, or following a delay of 1 month. Consistent with the findings of Steward et al. (1996), $11.3 \%$ of children reported at least one false instance of genital touch, and $25.5 \%$ of children reported at least one false instance of touch to the breast area (Willcock et al., 2006). Their subsequent study had similar findings with $7.1 \%$ of children reporting at least one instance of false touch to the genital area, and $23.8 \%$ of children reporting at least one instance of false touch to the breast area (Willcock et al., 2006). In a second laboratory based study Brown, Pipe, Lewis, Lamb and Orbach (2007) reported that HFDs elicited more false reports of touch than verbal only interviewing. A follow up study examining children's reports in a second interview after a 7 month delay, showed children interviewed with a HFD reported more information that children interviewed verbally only, but also made more errors (Brown, Pipe, Lewis, Lamb \& Orbach, 2011). Bruck (2009) found that following the introduction of HFDs children failed to produce any additional accurate information, and further, that they elicited inaccurate information when used to probe for information the child had already been asked about.

Studies so far have demonstrated not only that HFDs may elicit inaccurate information, but also that they may fail to support complete recall and reporting of experiences. This is illustrated by studies that have found children to have low reporting of any instances of touch at all, regardless of the condition under which they were interviewed. Brown et al. found that children's spontaneous reports of touch 
information were rare following one month (2007), and seven month (2011) delays between the target event and the interview. Bruck (2009) also reported that in general children had poor recall of touch instances, regardless of the condition they were interviewed under. Willcock et al. (2006) interviewed children either immediately following a target event, after 24 hours or after 1 month. Even immediately following the target even children reported less than $50 \%$ of scripted touches (Willcock et al., 2006). Most recently, Salmon, Pipe, Malloy and Mackay (2011) found that children reported only about one third of the total possible touch related information when interviewed 7-10 days after their target event.

A low rate of correct reporting of scripted touches across all of these studies suggests that instances of touch may not have been encoded in the first place to allow for subsequent recall. In each of these studies the scripted touches have appeared added on to the activity the children are engaging in. For example scripted touch has been examined in the context of a visit to the fire station (Willcock et al., 2006), a visit with a pirate (Brown et al., 2007; 2011; Salmon et al., 2011), or in the context of a magic show (Bruck, 2009). Research has demonstrated that peripheral details to an event are less well recalled compared with details that are more central to an experience (Klemfuss \& Ceci, 2009). This affects both the amount and the accuracy of information that is reported. As such, research has yet to establish the efficacy of HFDs when touch is a central feature of the event, in that it defines the actions that are occurring, as is the case in child sexual abuse.

In order to test the efficacy of visual recall aids it is essential that the instances of touch are encoded to begin with. While ensuring that the context of the study remains ethically safe by investigating innocuous instances of touch, an event more analogous to child sexual abuse instances where touch is the event needs to be 
established. The current study proposes to develop the methodology of recall aid research by utilising an event where touch is intrinsic to the staged event. We propose a target event themed around health and safety, incorporating instances of touch such as children having their temperatures measured and heartbeats listened to.

Across the 7 studies in which the effects of HFDs are examined there are a number of methodological differences and limitations, which the current study aims to address. Aldridge et al. (2004) compared information elicited in the verbal portion of the interview with information elicited in the portion of the interview following introduction of a HFD. They did not include a control condition of a verbal only comparison for the portion of the interview conducted after introduction of the HFD. As such it is difficult to establish whether the presentation of the HFD elicited further information because it served as a second opportunity to recall information or because of some inherent feature of the HFD. While Bruck (2009) presented a HFD either prior to or following a verbal interview, a verbal only comparison in place of HFD presentation was not included. To control for the possibility that providing a second opportunity to recall alone produces additional information, and to examine the nature of information elicited from interviewing incorporating no visual aid use, the current study will include a verbal only comparison condition to conditions incorporating visual aids.

Willcock et al., (2006) concluded that HFDs should not be used with 5-6 year old children due to their finding that $47 \%$ of reported touches following the introduction of a HFD were incorrect. They attributed this to children's failure to understanding the representative nature of the HFDs. Willcock et al. (2006) utilised a clothed 'gingerbread' or cartoon body map. Brown et al. (2007) included a verbal only control group and used a more realistic gender neutral unclothed HFD. They also 
reported HFDs elicited more false reports than verbal only interviewing. The current study utilises the unclothed non-anatomically detailed human figure diagram in Aldridge et al. (2004) commonly used during forensic interviews. This figure diagram allows for great precision in identifying body parts, for example in defining genital contact, and provides a better analogy to the forensic setting in which they are used. It may also reduce the cognitive load on the child of utilising an abstract body map such as the cartoon like figure the Willcock et al. (2006) study adopts.

Given that Brown et al. (2007) reported unsuccessful HFD use with a less abstract diagram, the current study will also compare HFD use with use of photographs of the children themselves. Presenting photographs in the place of HFDs of any kind should significantly decrease the cognitive demand of symbolic representation involved in HFD use, if this is an issue. While potentially addressing symbolic representation difficulties and providing a more effective retrieval cue, photograph use will also continue to provide the same opportunity for children to respond non-verbally to touch enquiry as HFDs do. This means that like HFD use, photographs should continue to address the potential limitations of verbal only interviewing, by assisting with language limitations and providing a non-verbal means of communicating potentially shameful or embarrassing information.

Anderson et al.'s study of New Zealand women highlighted 8 to 12 year old children as most at risk to being exposed to some form of sexual abuse (1993). Despite this, to date all of the studies examining the use of HFDs have been with children aged between 3 and 7 years, with the exception of a field study by Aldridge et al. (2004). This is presumably because of concern that children of this age will require the most support in recalling and reporting experiences. While children in this age group do undergo forensic interviewing, it is those who are over 7 years of age 
who are more likely to become involved in court processes. For these reasons it is essential that the most effective and safe ways to enhance recall and reporting of experiences are investigated in this older, at risk, age group. Teoh et al., (2010) who analysed the transcripts from the field study by Aldridge et al., (2004) argued that HFDs appeared to be more helpful for older, rather than younger children, in elaborating upon already reported instances of touch. While young children may use HFDs as a means of compensating for limited verbal language abilities, older children may find HFDs useful for completely different reasons. As children approach puberty they develop an increased awareness of their own bodies and become aware of negative social evaluations around the kinds of touch they may have experienced with sexual abuse. In this age group, visual aids may provide a non-verbal means of communicating detailed information that children feel uncomfortable talking about. In addition, older children are still developing their memory capacities and are still likely to benefit from support with retrieval of information in the same way that younger children are thought to. The present study proposes to investigate the effect of visual aids on recall and reporting of information in older children than have been previously studied, in children aged 9 to 11 years.

The present study will address several questions. First we will examine a broader age range than has previously been incorporated in HFD research, by including 9-11 year old children in our research sample. We expect that older children will still benefit from visual aids relative to verbal only interviewing. Second we will investigate the effect of the timing of introduction of visual aids and the purpose of their use, on the amount and accuracy of information that they elicit. We will examine this by introducing visual aids first for the purpose of elaborating upon or clarifying previously reported touch information, followed by for the purpose of eliciting new 
touch information. We expect information to be more accurate when visual aids are used for elaboration or clarification of already reported touch information, relative to when they are used for the purpose of eliciting new touch information. Third we will examine the effect of a new event methodology on the nature of touch information that is recalled, by incorporating instances of touch as a central feature of the target event. Relative to previous studies where touch was added on to the main theme of the event, we expect a higher recall rate of scripted touches. Fourth we will explore a new non-verbal interviewing technique by introducing photographs of the children as compared with a HFD or verbal only prompts. As this is an exploratory study we make no predictions regarding the effect of photographs on the nature of information that is elicited during interviewing.

\section{Method}

\section{Participants}

One hundred and thirty children from six class rooms experienced the staged event. Children run through the staged event were excluded from interviewing for the following reasons; the children did not experience the event in a standardised manner due to uncontrollable circumstances, parental permission was not obtained, children were absent from school during the interviewing period. Ninety of the children who experienced the event were interviewed. Interviewed children were excluded from the final sample for the following reasons; children were recruited from an older year group, however there were insufficient numbers to include as a separate age bracket ( $n=18)$, audio files were of insufficient quality to be transcribed $(n=16)$, children clearly had a limited understanding of English which significantly inhibited their 
ability to communicate during the interview $(n=1)$. Data collection took place between March and September 2011.

The final sample was comprised of 55 children (26 males and 29 females) aged between 9 and 11 years (mean age 10.2 years) recruited from two public schools within the Wellington region. The study was approved by the Victoria University School of Psychology Human Ethics Committee, and agreement was obtained from the principals of the schools involved. Written consent was obtained from the parents/caregivers of each child (see Appendix A and B), and verbal assent was obtained from each child prior to commencement of both the event and the interview. Each child received a small gift (e.g. a novelty pencil) in recognition of their participation.

Design

A repeated measures between-participants experimental design was used, with structured interviews conducted after a delay of approximately two weeks from the time of the staged event. Interviews followed an ecologically valid forensic protocol adapted for experimental use (Orbach et al., 2000). The quantity and accuracy of information concerning bodily contact that was recalled and reported about the event was measured. The way in which information about touch was elicited following a verbal interview was assessed through three conditions (between-participants); verbal questioning alone, HFDs, and a photograph of the child. The amount and quality of information reported during the three phases of interview (verbal interview, follow-up questions, specific touch questions) was examined as a within-participants factor.

\section{Materials}

Event. Props were used to stage a 'health and safety' themed event. Props involved in the event included stethoscopes, two different types of thermometer, a 
temperature chart on which temperatures were recorded, red felt-tip pens, antiseptic wipes, sticking plasters, a brief video clip and power point presentation played on a lap top computer, and picture cards demonstrating hazardous situations.

Interview. During some interviews gender-neutral, unclothed HFDs with no anatomical detail (see Appendix C), or photographs of the children themselves, taken with a digital camera as they entered the interview and printed using a portable colour printer, were used. During interview conditions where a visual aid was used coloured 'dot' stickers were applied to HFDs and photographs as indications of where bodily contact occurred. Interviewers all followed the same interview script (see Appendix D). This was used as a guide to maintain consistency between interviewers, but was not expected to be followed verbatim, given the child-led flexible nature of the interview protocol. Monitor sheets (see Appendix E) were used during the interview phase to guide the monitor in checking off the main aspects of the event as the child reported them. It was also used for the monitor to record any information the child gave that was either ambiguous and required clarification, or lacked in detail, to assist them in providing recommendations to the interviewer during the monitor's break.

\section{Procedure}

Target event. Children took part in a class-based event lasting approximately 40-50 minutes, where they rotated through four stations led by research assistants, learning about different aspects of health and safety. The children were placed into groups of 4-8 and rotated around the stations in these groups. There was an additional research assistant present who introduced the event and indicated when the groups should move to the next station. The four stations included identifying hazards, looking after cuts, listening to heartbeats, and measuring temperatures. At the hazards activity children were shown a number of picture cards depicting hazardous situations 
and asked to identify them. At the cuts activity children were shown a video clip of a cartoon character having an accident involving a cut, and were then shown a power point presentation of how to care for a small cut. They were instructed to draw a red line on their finger to simulate a cut and then follow along with the power point in applying pressure and raising it above their heart, wiping it with an antiseptic wipe and applying a plaster. Children were then asked to gather in a group and hold up their finger, and a photograph was taken of them. At the heartbeat activity children were given a brief talk about the function of the heart, and were then instructed to get into pairs and listen to their partner's heartbeat using a stethoscope in a number of places; the chest, the back and the stomach. They were then instructed to feel for their partner's pulse in a number of places; the wrist, the neck and the ankle. At the temperature activity the children were introduced to the concept of temperature as an indication of wellness. They were then instructed to get into pairs and measure their partner's temperature in a number of places; the forehead, the ear, the armpit and behind the knee, and record it on a temperature chart. Two of the activities incorporated instances of innocuous bodily contact between participants. Some of this bodily contact was normal or expected (eg. having their heartbeat measured on their chest), and some of it was unusual or unexpected (eg. having their heartbeat measured on their stomach). In total there were ten instances of bodily contact (see Table 1). The staged event was videotaped for the purpose of checking accuracy of details reported during the interviews. 
Table 1. Instances of touch in scripted event

\begin{tabular}{lll}
\hline Activity & Script-related touch & Unexpected touch \\
\hline Temperature & Armpit & Knee \\
Ear & \\
Forehead & \\
Heartbeat & Chest & \\
& Back & Stomach \\
& Wrist & Ankle \\
Neck & \\
\hline
\end{tabular}

Event interview. Approximately 2 weeks later children were interviewed by an unfamiliar research assistant under one of three conditions; verbal only prompts, verbal prompts and HFD, or verbal prompts and photograph. The interview followed the National Institute for Child Health and Development (NICHD) Investigative Interview protocol, modified for experimental use (Orbach et al., 2000). The initial part of the interview consisted of establishing interview ground rules with the participant. These included telling the truth, saying I don't know or I don't understand where necessary, and correcting the interviewer when they make a mistake. Next an opportunity to practise responses to the types of prompts likely to be encountered in the interview, and to practise reporting a recent past event, was provided by asking children about what they had done during the day. During the section of the interview targeted at recall of the event, a scripted prompt was used to orient the children to the health and safety event. Interviewers then encouraged children to provide elaborative accounts of the event, using a range of different 
prompts. As the NICHD protocol is a flexible protocol, the order and frequency of these varied across interviews, however interviewers were encouraged to give priority to the prompts as follows; Invitations: Invitations are utterances prompting free-recall responses from the child e.g. "tell me all about that", or "tell me more about that". Cued invitations: Cued invitations use details already disclosed by the child as prompts for further elaboration e.g. "you mentioned that at the heart beat station you were measuring heart beat with your partner, tell me everything about that". Directive prompts: Directive prompts refocus the child's attention on details of the incident of interest that the child has already mentioned, and using Wh- questions, they provide categories for requesting additional information e.g. "you mentioned that your partner listened for your heart beat, where did they listen for your heart beat?". Option posing prompts: Option posing prompts focus the child's attention on aspects related to the incident of interest that the child has not previously mentioned, asking the child to affirm, negate or select an interviewer-given option, without implying that a particular response is expected e.g. "did your partner touch you on the knee?"

In the event recall section exhaustive open-ended invitation was employed (e.g. "tell me more about that") until children reported that they could recall no more information. Following this cued invitations using information already provided by the child were used to elicit further detail about reported incidents (e.g. "you said you measured heartbeats; tell me all about that"). These prompts used the child's exact wording. Directive prompts were then used only if needed for clarification or detail not revealed by exhaustive open-ended questioning (e.g. "you said you were in partners, who was your partner?"). At this point the interviewer took a short break where they consulted with a monitor to see whether any further questioning needed to occur. 
The monitor was another unfamiliar research assistant trained in the interview protocol who was also present in the room during the interview. The role of the monitor was to use the monitor sheet (see materials section) to record aspects of the scripted event that had been reported, and to note down any information the child verbalised that was ambiguous or lacked in sufficient detail. They then, during an interval in the middle of the interview, prompted the interviewer to follow up on any information provided by the child that was ambiguous or lacked in sufficient detail. The purpose of having a monitor was to ensure that interview protocol was followed and thus increase the consistency of interviewing across children, and to ensure that interviewers followed up on verbalisations from children that were unclear thus eliciting as much information as possible about their experience. The use of a monitor in this manner is also analogous to current forensic interviewing practise in New Zealand.

At the conclusion of this section of the interview, the touch enquiry section of the interview commenced and was conducted in one of the three ways described below. Interviews lasted approximately 30 minutes and were videotaped for transcription and analysis.

Touch enquiry

\section{Verbal only}

Touch follow up interview phase. In the verbal only condition participants were asked to verbally reiterate each individual instance of already reported touch (e.g. "you told me that your partner touched you on the wrist at the heart station, tell me exactly on your body where that happened"). If any new incidences of touch were recalled during this phase children were asked to elaborate using an open-ended prompt. 
Specific touch questions interview phase. In this interview phase, participants were asked a series of questions about unreported touch using option posing prompts. These questions included any instances of touch that did occur during the event but that were not reported during the initial interview. They also included six false questions; three instances of touch that did not occur (e.g. "did your partner touch you on the mouth?"), and three instances of touch that did occur but were about a partner that the child did not work with (see Appendix D for list of questions). If questioning elicited a 'yes' answer, children were invited to elaborate on the report using an openended prompt (e.g. "tell me all about that"). If children responded "no" to a question of an instance of touch, the question was then repeated with the inclusion of an action prompt (e.g. "did you partner put a thermometer in your mouth?”). Again if children provided a 'yes' answer, they were invited to elaborate on the report. There were 16 specific touch questions in total, but the actual number of questions that were posed to each child depended upon whether they had already reported the touch in the previous phases of the interview.

\section{Human figure diagram}

Touch follow up interview phase. In this condition children were presented with a human figure diagram depicting both front and back views of a body and asked to place a red sticker on the diagram to show where on their body each previously reported incidence of touch occurred. If any new incidences of touch were recalled during this phase children were asked to elaborate using an open-ended prompt, and were asked to indicate where it occurred on the human figure diagram with a red sticker.

Specific touch questions interview phase. Following this participants were asked the same series of questions about unreported touch as the verbal interview 
group. If they responded 'yes' in response to any of these questions they were asked to place a yellow sticker on the human figure diagram to indicate where on their body the touch occurred. Again if children provided a 'yes' answer, they were invited to elaborate on the report, and place a sticker on the human figure diagram to indicate where on their body it occurred.

\section{Photograph}

In this condition children were presented with a photograph of them that had been taken at the beginning of the interview depicting both front and back views and printed using a portable colour printer. The procedure was then identical to that of the Human Figure Diagram group.

\section{Coding}

All interviews were transcribed including verbal and non-verbal responses. Non-verbal responses included the child pointing to them self or the visual aid. Verbal and non-verbal instances of reported touch were coded according to the type of question that elicited the response, and the section of the interview that it was elicited in; initial verbal interview, touch follow-up interview, or specific touch questions. Data was also coded according to the accuracy of the touch reported, and the quantity of touch reported. Units of meaningful touch-related information were categorised as correct, incorrect distortion, incorrect intrusion, prompted correct, prompted incorrect distortion, prompted incorrect intrusion, or other. Incorrect distortion was used to code instances of reported touch that did occur but not in the way the child reported it, for example, 'he put the stethoscope on my wrist' when the child's partner actually put his fingers on the child's wrist. Incorrect intrusion was used to code for instances of reported touch that did not occur at all, for example, 'she put the thermometer in my mouth' where this was not part of the event. Other was used to code subjective 
(e.g. 'it felt weird') or ambiguous (e.g. 'he put the thingy under') touch information that was unverifiable. Prompted codes were used for instances of touch that were elicited in response to information provided by the interviewer that the child had not previously reported, for example a 'yes' response to the question 'did your partner touch you on the knee?' when the child had not previously reported being touched on the knee. Repeated information was acknowledged on the transcript to assist with reliability coding but was not included in analysis.

Coding procedure. A coding manual was developed (see Appendix F) defining each code and providing examples of when this code should be applied which were extracted from the study transcripts. The beginning of each phase of the interview was identified. All touch related information was identified. To establish the proportion of reported touches and their accuracy, each touch that was reported was tallied according to the phase of the interview that it was first elicited (verbal, phase one of the touch interview, the touch specific interview), and was defined as correct, incorrect distortion or incorrect intrusion. To establish the accuracy of the touch related information reported during the specific questioning phase, the number of specific questions that were posed to the child were recorded, and their answers were defined as correct, incorrect distortion, incorrect intrusion or don't know. This was recording on a coding sheet (see Appendix F). To establish the quantity of touch information reported, each new touch related detail was then coded according to the criteria outlined in the above section. Instances of touch related details were then tallied according to their code on a second coding sheet (see Appendix F).

\section{Reliability}

A second coder coded $20 \%$ of the transcripts. The second coder was trained using the following protocol; they were provided with the coding manual, they 
observed the primary coder code one transcript, they collaboratively coded two transcripts with the primary coder, they coded five transcripts independently and were provided with feedback by the primary coder (these were not included within the final reliability sample). Percentage agreement (75\%) was calculated to determine intercoder reliability on the final reliability sample. The data generated from the primary coder was used for all analyses.

\section{Analysis}

Repeated measures between subjects analysis of variance (ANOVA) were used to analyse instances and accuracy of reported touch within the verbal interview phase, and within the follow up touch and specific touch interview phases combined, according to the condition under which it was reported (verbal only vs. HDF vs. photograph). Proportion of scripted touches reported was examined using univariate ANOVA analyses. Instances of intrusions were examined using chi square analyses.

\section{Results}

We examined the proportion of scripted touches reported across the total sample. We then examined the nature (amount and accuracy) of the touch related information that was reported. Firstly, we examined the nature of the touch-related information elicited during the verbal interview phase, prior to experimenter manipulation. This allowed for consideration of how well children had encoded and were able to spontaneously report instances of touch. Secondly, we examined the nature of the information elicited during the follow up touch and specific touch questions phases of the interview, following increased interviewer input and the introduction of the experimental manipulation. Amount and accuracy of information was examined as a function of the phase of the interview in which it was elicited 
(touch follow up vs specific questions) across interview conditions (HFD vs photograph vs verbal only). We also examined instances of incorrect intrusions reported following introduction of the experimental manipulation as a function of the phase of the interview in which it was elicited (touch follow up vs specific questions) across interview conditions (HFD vs photograph vs verbal only).

Proportion of scripted touches reported was calculated as number of correctly reported scripted touches/(total number of scripted touches; $n=10)$. Total number of touch-related details (amount of information) reported for each phase of the interview (verbal, touch follow up, specific question) was calculated as the sum of all touchrelated information (verbal and non-verbal) reported in that phase collapsed across the codes of correct, incorrect distortion, incorrect intrusion, prompted correct, prompted incorrect distortion, prompted incorrect intrusion, other and don't know. Total accuracy of information reported for each interview phase (verbal, touch follow up, specific question) was calculated as correct information/total information. Instances of incorrect intrusions were examined categorically as being either present or not present due to low instances of intrusions overall.

Where measures were proportions, they were subjected to arcsine transformations. Raw means and standard deviations are reported in Table 2 and Table 3. Effect sizes are reported when tests were significant, and a measure of observed power is reported when tests were not significant. Partial eta-squared $\left(\eta_{\mathrm{p}}{ }^{2}\right)$ was used as a measure of effect size, with .10 indicating a small effect, .30 a medium effect and .50 a large effect (Field, 2009). Observed power $(\beta)$ measures the probability of failing to detect an effect when one genuinely exists (Type II error), and should be at or above .80 to detect an effect (Field, 2009). An alpha level of .05 was used for all statistical tests. Data was examined for normalcy of distribution and 
outliers. Outliers were corrected by 2 standard deviations above or below the mean according to Field (2009).

\section{Preliminary analysis}

Preliminary analyses of the data, conducted using analyses of variance (ANOVAs), revealed no main effect of gender, school or interviewer on amount or accuracy of touch-related information reported. Pearson's correlation revealed no significant relationship between age and amount or accuracy of touch relatedinformation reported. These factors were therefore not considered in subsequent analyses.

\section{Reporting of scripted touches}

Scripted touches were examined by considering the proportion of touches reported throughout the entire interview. The touches experienced in the event were overall frequently recalled and reported with the mean proportion of touches reported across all conditions being $0.87(S D=.11)$, or $87 \%$. A univariate ANOVA revealed no effect of condition on the proportion of scripted touches that were reported $F(2,52)=1.61, p=.21, \beta=.33$ (Table 2$)$.

\section{Spontaneous recall and reporting of touch-related information}

Analyses were conducted to examine children's spontaneous reporting of touch-related information during the verbal phase of the interview (i.e., prior to the introduction of interviewer-led strategies to elicit further detail). Univariate ANOVA analyses revealed no main effect of interview condition on amount $F(2,52)=1.17$, $p=0.32, \beta=.25$, or accuracy $F(2,52)=.47, p=.63, \beta=.12$, of information reported within the verbal interview phase (see Table 2). To examine the relationship between the amount of information children reported and the accuracy of their reports, 
Pearson's correlations were conducted; these indicated no significant relationship between accuracy and amount of touch-related information for each separate interview condition and across the whole sample.

Table 2. Mean $(S D)$ number of details and accuracy, and proportion of scripted touches reported by children during the verbal interview.

Phase 1

\begin{tabular}{|c|c|c|c|}
\hline Interview condition & $\underline{\text { Amount }}$ & Accuracy & $\frac{\text { Proportion }}{\text { (across entire interview) }}$ \\
\hline Photograph & $28.40(7.60)$ & $.86(.12)$ & $.90(.09)$ \\
\hline HFD & 28.35 (6.69) & $.86(.11)$ & $.83(.12)$ \\
\hline Verbal & $31.56(7.25)$ & $.87(.12)$ & $.87(.12)$ \\
\hline Total & $29.42(7.24)$ & $.87(.12)$ & $.87(.12)$ \\
\hline
\end{tabular}

Reporting of touch-related information following introduction of visual aids

Amount. Analyses were conducted to examine the amount of touch related information reported following experimenter manipulation (being presented with a HFD, photograph, or focussed verbal questions) across the follow up touch and specific touch questions phases of the interview.

Repeated measures ANOVA analyses revealed no main effect of phase on amount of information elicited $F(1,52)=.31, p=.58, \beta=.09$.

A significant main effect of condition on the amount of information elicited was observed $F(2,52)=3.30, p<.05, \eta_{\mathrm{p}}{ }^{2}=.11$. Tukey tests revealed children in the HFD condition reported significantly more information than the verbal condition, with children in the photograph condition interim between the two (see Table 3). 
Table 3. Mean $(S D)$ number of details and accuracy of touch information reported during phases 2 and 3 of the interview.

\begin{tabular}{llll}
\hline Condition & Phase & Amount & Accuracy \\
\hline Photo & 2 & $8.20(4.12)$ & $.70(.33)$ \\
& 3 & $8.10(3.82)$ & $.25(.21)$ \\
HFD & 2 & $9.88(4.15)$ & $.76(.28)$ \\
& 3 & $7.97(4.19)$ & $.28(.26)$ \\
Verbal & 2 & $4.72(4.57)$ & $.77(.26)$ \\
& 3 & $8.28(5.74)$ & $.29(.26)$ \\
Total & 2 & $7.58(4.71)$ & $.74(.30)$ \\
& 3 & $8.12(4.55)$ & $.27(.23)$ \\
\hline
\end{tabular}

A phase $\mathrm{x}$ condition interaction approached significance $F(2,52)=2.93, p=.06$, $\beta=.55)$. Univariate ANOVA analyses were conducted separately for the touch follow up and touch specific questions phases of the interview to examine the amount of information elicited as a function of interview condition. Analyses revealed a significant main effect of condition on amount of information elicited in phase two of the interview (touch follow up) $F(2,52)=6.67, p<.01, \eta_{\mathrm{p}}{ }^{2}=.20$. Tukey tests revealed that in phase two of the interview, both the HFD and the photograph conditions elicited significantly more touch related information than the verbal only condition, but did not differ significantly from each other (see Table 3). No main effect of 
interview condition on amount of information elicited in the touch specific questions phase of the interview was revealed $F(2,52)=.02 . p=.98, \beta=.05$. (see Figure 1).

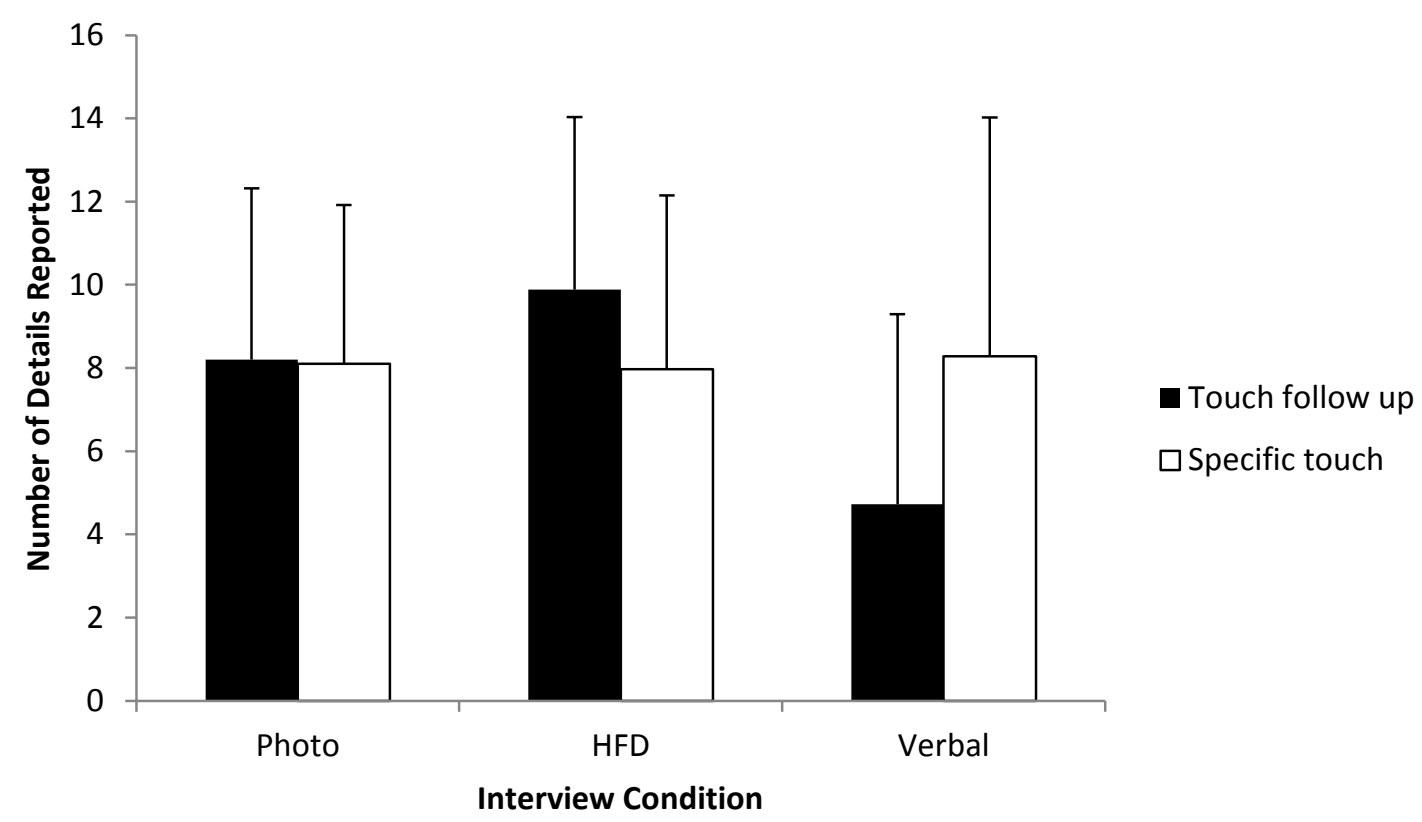

Figure 1. Amount of information elicited in touch follow up (phase 2) and specific touch questions (phase 3) phases of the interview by condition.

Accuracy. Analyses were conducted to examine the accuracy of touch related information reported following experimenter manipulation (being presented with a HFD, photograph, or focussed verbal questions) across the follow up touch and specific touch questions phases of the interview.

Repeated measures ANOVA analyses revealed a significant main effect of phase on the accuracy of touch-related information reported $F(1,49)=91.16, p<.001$, $\eta_{\mathrm{p}}{ }^{2}=.65$. The follow up touch phase elicited significantly more accurate information than did the specific touch questions phase of the interview (see Table 3.) 
No main effect of condition on accuracy of information elicited was revealed $F(2,49)=.25, p=.78, \beta=.09$ and no interaction was observed $F(2,49)=.01, p=.99, \beta$ $=.05$. (see Figure 2.)

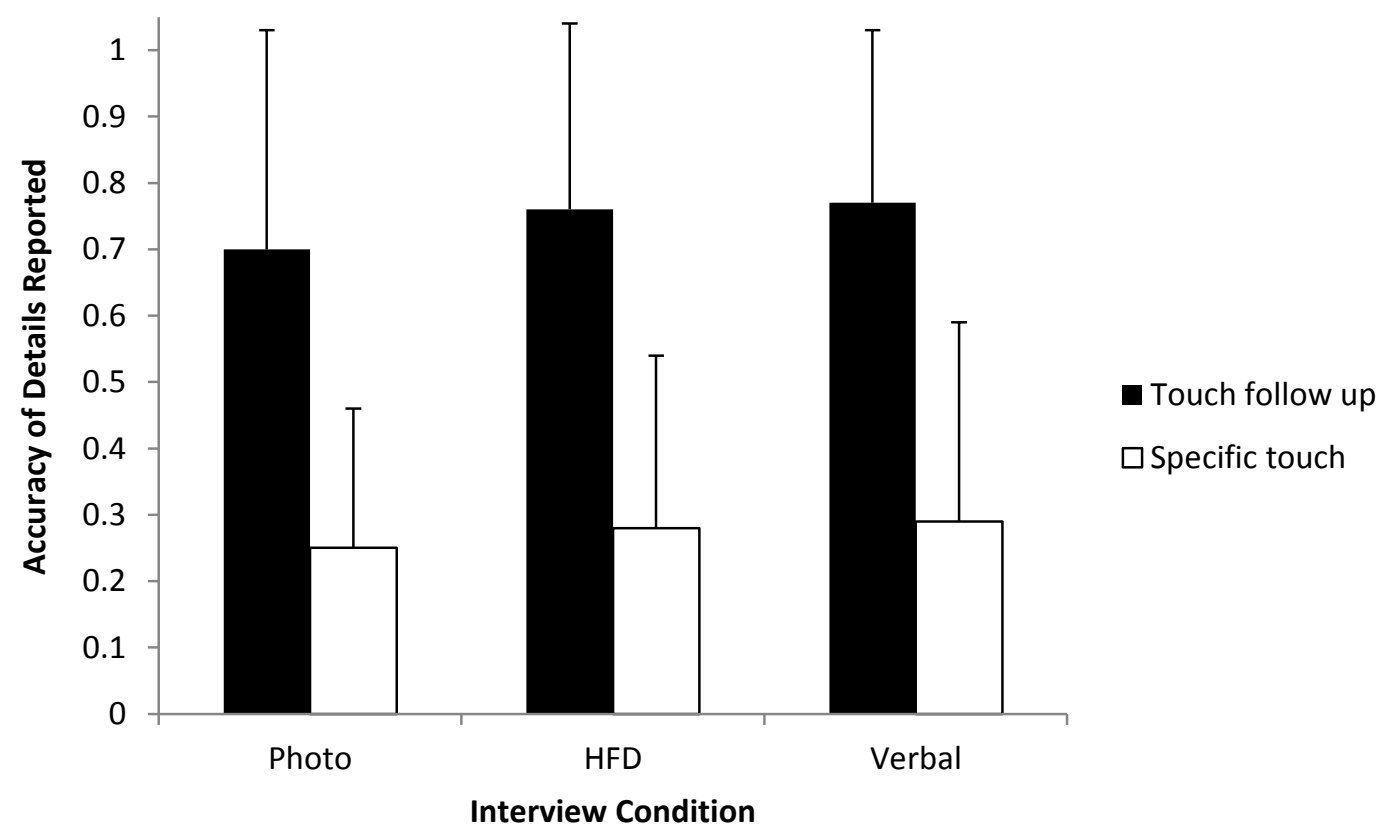

Figure 2. Accuracy of information elicited in touch follow up (phase 2) and specific touch questions (phase 3) phases of the interview by condition.

\section{Intrusions}

Intrusions were not examined for the verbal only phase of the interview as this phase involved no experimenter manipulation. Intrusions were rare, with only 5 of the 55 , or $9 \%$, of children reporting any touch intrusions in the follow-up or specific touch questions phases of the interview. As such intrusions were coded as present or absent. Chi square analyses revealed that whether children made intrusions or not did not differ as a function of how they were interviewed, $x^{2}(2)=2.18, p=.34$. 


\section{Discussion}

\section{Reporting of scripted touches}

We examined the effect of a new target event developed to incorporate instances of touch central to the event on the nature of touch information reported. Our hypothesis was that children would recall and report a higher proportion of scripted touches than in previous literature, where touch appeared more peripheral to the nature of the target event. On average ${ }_{2}$ children in our study recalled and reported $87 \%$ of scripted touches. This is in contrast to findings in previous studies where spontaneous recall of touch was infrequent (Brown et al. 2007; 2011; Bruck 2009; Willcock et al., 2006; Salmon et al., 2011). The target event adopted in the current study aimed to incorporate instances of touch central to the theme of the activity i.e. a health theme included touch instances of having temperature measured and heartbeat listened to. This is in contrast to previous studies where scripted touches were added on to the potentially more memorable target event e.g. touch instances while dressing in a fireman's outfit before visiting a fire station. As a result, children may not have been encoding the information to begin with, and thus, were unable to report it. The finding of the current study suggests that instances of touch were better encoded and children were more readily able to recall and report them. An alternative consideration is that the current study employs an older age group of children (9-11 years) than many of the previous studies who examined reports in 3-7 year old children (Brown et al., 2011; Brown et al., 2007; Willcock et al., 2006; Bruck, 2009). It is possible that older children are simply better able to encode, recall and report instances of touch than younger populations. Future research should seek to investigate the effect of age using the event methodology adopted by the current study to evaluate the efficacy of the protocol on younger children. Nonetheless, the current 
findings demonstrate an increased capacity for researchers' to study the effects of experimental manipulations on children's recall and reporting of touch information.

\section{Spontaneous recall and reporting of touch-related information amount and accuracy}

Within the verbal phase of the interview, we detected no main effect of condition on the amount or accuracy of touch-related information reported, indicating no significant differences in baseline amount or accuracy of reported touch-related information. Given that the verbal phase of the interview was not subject to any experimenter manipulation, our findings suggest that any significant differences detected following implementation of experimenter manipulation are likely to be in response to the manipulation as opposed to individual differences in reporting. The number of touch related details reported in the verbal interview approached 30, and of these $87 \%$ of details were accurate. This finding continues to demonstrate support for the NICHD investigative interviewing protocol (Orbach et al., 2000) which specifies free recall prompts should be utilised exhaustively as a first port of call given their established propensity to produce the greatest quantity and the most accurate information in children's reports of their experiences. Furthermore, the accuracy of the children's reports demonstrates that, when the touches are salient and children are interviewed relatively recently after their experience, they are able to provide detailed descriptions of what happened to them without intensive input from the interviewer. The improved accuracy of the information reported, relative to previous studies, may once again reflect the encoding strength of the children's memories. Previous studies of children's memory have typically demonstrated that while the amount of detail children report increases with age, the accuracy of that information remains constant, at least when free recall is considered (Pipe et al, 2004). As such, the heightened accuracy in our study is not likely to be simply a product of the older sample, and 
provides more evidence for the utility of our event as a useful context from which to study children's reports of touch.

The relationship between the amount and the accuracy of information reported

Our analyses revealed no significant relationship between the amount and the accuracy of information reported when examined across interview conditions.

Therefore, at least for the current age group, eliciting a greater amount of information does not appear to lead to a decline in the accuracy of children's reports. We also examined the relationship between amount of information elicited and accuracy individually for each interview condition. Previous studies have demonstrated HFD use to elicit more information, but also more erroneous information when compared with verbal interviewing alone (Steward et al., 1996; Brown et al., 2007; Brown et al., 2011). In contrast, our findings revealed no relationship between amount of information elicited and the accuracy of this information for any interview condition. This is inclusive of our two interview conditions that utilise visual aids, following the rationale that they may elicit more touch related information than verbal only interviewing (Pipe \& Salmon, 2009). Therefore, even with the use of currently controversial visual aids, we have demonstrated that at least for the current age group, when additional information is reported it does not appear to be at the expense of the accuracy of that information.

\section{Reporting of touch-related information following introduction of visual aids}

Amount. Children presented with a HFD reported significantly more touchrelated information than children in the verbal only condition, with the photograph condition interim between the two. This is consistent with previous literature demonstrating that visual aids elicited a greater amount of touch related information relative to verbal only interviewing (Brown et al., 2007; Brown et al., 2011; Steward 
et al., 1996). We aimed to examine the effect of HFD use on a broader age group of children by incorporating older children (9-11 years) than had previously been studied. We expected that older children would still benefit from HFD use relative to verbal only interviewing, despite their potentially enhanced capacity for reporting their experiences compared with younger children. Our results indicate that when children aged 9-11 are presented with a HFD they report more touch related information than when they are interviewed with verbal only prompts.

The amount of information reported during the two "intervention phases" of the interview did not differ as interviewer input increased. One possibility is that children in this older age bracket are generally more competent in being able to recall and report their experiences through initial verbal free-recall techniques. We found children to report on average almost 30 touch related details during the verbal portion of the interview. In contrast they reported an average of approximately eight touch related details in the touch follow up phase of the interview and an additional eight touch related details in the specific questioning phase of the interview. It is possible that we are observing a ceiling effect where the majority of touch related information has been elicited during the verbal portion of the interview and there is limited touch related information remaining to be recalled and reported during the phases of the interview following visual aid presentation. However, it may also demonstrate support for our target event and imply that because touch information was so well recalled, it was well encoded to begin with. Given the nature of forensic interviewing any touch information that is reported is vital. Therefore, if a visual aid has the capacity to produce any new touch information at all (provided it is accurate information) then its use should be considered. 
There were indications that the way in which children were interviewed during different phases of the interview influenced their recall, such that visual aids elicited more information than verbal questioning alone during the follow-up phase of the interview. Whilst we must interpret this with caution, given the analysis reflected a trend rather than a significant difference, this is an important finding that should be replicated with a larger sample size.

We aimed to examine the effect of a new non-verbal interviewing technique (a photograph of the child) on the amount and accuracy of the information children reported. No significant difference was detected in the amount of information elicited following introduction of an HFD compared with a photograph. It is possible that our sample of older children may not have difficulty effectively understanding and utilising symbolic representations. This may have meant that being presented with a photograph (with less need to understand symbolic representation) was no more effective in eliciting additional touch information than being presented with an HFD (with a higher symbolic interpretation demand). Previous studies demonstrate that as children get older ${ }_{2}$ they are more likely to be able to effectively understand and utilise symbolic representations of themselves, whereas very young children may struggle with this (DeLoache 2000; 2004; Salmon, 2001). Future research should seek to compare the current age group with a younger population to determine any differences in amount of information reported following introduction of HFDs compared with photographs. This would help determine whether difficulties interpreting HFDs as symbolic representations do in fact contribute to young children's inability to use them effectively, as has been previously suggested (Willcock et al., 2006). 
In older children, the use of a visual aid regardless of its nature may serve as an effective external memory cue and elicit more touch-related information compared with verbal only interviewing. This is consistent with current rationale for utilising visual aids (Pipe \& Salmon, 2009). In their field study, Aldridge et al. (2004) incorporated children up to 13 years of age and demonstrated HFDs to elicit a significant number of additional forensically relevant details following those elicited during exhaustive verbal interviewing. In their examination of the transcripts from this study, Teoh et al. (2010) demonstrated that HFDs assisted older children in clarifying instances of already reported touch. We also demonstrated that the HFDs were particularly effective in assisting children to clarify previously reported information. That is, the effects of the visual aids were apparent during the follow-up phase of the interview where children were asked to clarify and/or elaborate upon information they had reported earlier. During the specific touch questions phase where children were asked about previously unreported touch, no benefit of visual aids was evident. Visual aids may assist children to communicate their experiences by both reducing the cognitive demand on the child, and/or providing an effective external retrieval cue, thus potentially leading children to report an increased quantity of touch related information. The findings of the current study add to existing literature reporting that HFDs elicit a greater quantity of information than verbal only prompts, and do so with older children than previously studied. Furthermore, we demonstrated that a photograph of the child can be equally effective.

We did not observe an effect of interview condition on amount of information elicited in the specific questions phase of the interview. Firstly, as alluded to above, it may be that we are observing ceiling effects due to the current age group of children being able to recall and report information so well during the verbal interviewing 
phase of the interview that little information is left to be reported by the second and third opportunities to recall. Therefore, it may be difficult to observe any potential differences that could exist between conditions. Secondly, while visual aids are present during this interviewing phase, they are utilised only in the case that the child actually reports new touch related information that has previously not been reported. In this case, children would then be asked to indicate on the visual aid where the touch had occurred and be invited to elaborate upon it. While the visual aids are present the type of questioning that is largely endorsed in this phase of the interview is recognition prompting. When children are presented with these kinds of prompts it is possible that they are being asked to make decisions about information that they may not have encoded properly to begin with. We included instances of omitted touch as part of our measure of total touch information. Therefore, it may be that while the amount of information elicited in the specific touch phase of the interview was the same as that of the touch follow up phase, it may have also included more incorrect pieces of information (see below).

Accuracy. We examined the accuracy of the information elicited as a function of interview condition. We found no main effect of interview condition on accuracy of information reported. This indicates that, in contrast to previous literature (Brown et al., 2007; 2011; Steward et al., 1996; Willcock et al., 2006; Bruck, 2009), the introduction of HFDs did not elicit more erroneous reports in comparison to using verbal only techniques. In combination with our findings that visual aids (both photographs and HFDs) elicit a greater amount of touch related information than verbal only prompts, we are able to suggest that at least with children aged 9-11 it may be, not only safe, but also beneficial to utilise visual aids in forensic interviewing. 
We expected that the phase of the interview would impact upon the accuracy of the information elicited. Specifically, we hypothesised that the touch follow up phase of the interview would elicit more accurate information than the specific touch questions phase of the interview. This was based on established literature indicating that recall prompts such as those prioritised in the follow up phase of the interview elicit more accurate information than recognition prompts such as those prioritised in the specific touch questions phase of the interview (Lamb et al., 2007). Consistent with our predictions, information reported during the follow up interview phase was significantly more accurate than the specific touch questions interview phase. Specifically, accuracy of information in the touch specific questions phase of the interview was on average only $27 \%$, in contrast accuracy of information elicited in the touch follow up phase of the interview was on average $74 \%$. This indicates that children report mostly accurate information when interviewer input remains minimal and free-recall prompts are the major interviewing technique employed, such as in our touch follow up interview phase. Once interviews become interviewer rather than child led and recognition prompts increase, such as in our specific touch questions phase, the accuracy of reporting significantly decreases. One criticism of previous HFD research has been that it has examined their use in ways that may not mirror how they are used in forensic interviews (to elicit new information rather than to encourage elaboration of previously reported information) (Salmon et al, 2011). Our study was able to compare the two and showed that although further questioning did elicit new information, visual aids did not differentially elicit more information, and the accuracy of information across all conditions was poor. This suggests that visual aids may be beneficial, at least for older children, but only when asking about previously reported information. Consistent with previous studies (Brown et al., 2007; 
2011; Willcock et al., 2006; Steward et al., 1996; Bruck et al., 2009), even with older children, visual aids should not be used to elicit new information as the likelihood of eliciting false information is too great.

Our measure of accuracy incorporated not only instances of false reports, but also instances of omitted touch information i.e. when children were directly asked whether a specific scripted touch occurred, and they responded negatively, this was considered inaccurate touch information. This means that our figure of $27 \%$ accuracy in the touch specific questions interview phase incorporates a proportion of falsely unreported touches, as well as falsely reported touches. Literature thus far has been largely interested in instances of falsely reported touch as analogous to false accusations in sexual abuse cases. However, we would argue the erroneous omissions of touch information are equally as important as falsely reported instances as they are analogous to non-disclosure of sexual abuse, and consequently lack of protection for children from abuse. The implications from our findings are that, in line with current NICHD protocol, we strongly recommend that specific directive types of questioning relying on children's recognition memory are principally avoided in forensic interviewing.

\section{Intrusions}

We examined instances of intrusive false touch information elicited throughout the follow up touch and specific touch questions phases of the interview. Overall we found that children's reports of touch intrusions were rare, with only $9 \%$ of children falsely reporting instances of touch that did not occur at all. In addition, instances of reported touch intrusions did not differ significantly according to interview condition. This offers additional support for safe visual aid use with children at least of the current age group. However, due to the extremely small 
proportion of touch intrusions reported across all conditions, statistically it would be difficult to detect differences by condition if they were to exist. It is also possible that children of this age group are generally able to accurately report their experiences, in that they infrequently make reports of entirely false touch information. However, there is evidence that children are more likely to make errors of omission than errors of commission (Goodman \& Reed, 1986). Given that we found only 9\% of children to report touch intrusions, but also showed on average only $27 \%$ accuracy when reporting in the specific touch questions portion of our interview, it follows that errors in touch information elicited throughout the current study were more likely to be of omission than of commission. As this may be analogous to non-disclosure in reporting, it seems pertinent that we continue to consider methods of aiding children towards more complete and accurate recall and reporting of their experiences.

\section{Limitations}

The results of the current study must be interpreted with caution given the innocuous nature of the touch the children experienced. Children participated in a fun event, in a familiar environment. Instances of touch occurred in a manner that is publicly sanctioned and encouraged, to areas of the body that are not generally associated with embarrassment. It is worth noting however, that this does not make research of this type redundant for forensic interviewers. That is, whilst laboratory based research is inevitably limited in the extent to which it can ethically provide an analogue to forensic contexts, not all abusive touch is experienced by children as traumatic (or even salient). It is important that techniques that may be used in the forensic context are examined in a more controlled environment (e.g. laboratorybased studies) to determine their efficacy and any caveats associated with their use 
before they are introduced into investigative interviews, where they may exert potentially damaging effects on the lives of alleged victims or perpetrators.

The time delay between the children experiencing the target event and subsequently being interviewed was relatively short compared with typical forensic practises. While this represents a limitation, in that the passage of time elapsed was not analogous to delays that may typically (but not always) occur in forensic interviewing, it is an important step towards establishing an effective method of studying forensic interviewing techniques with children. This was a particularly salient issue for the current study given that we aimed to establish the usefulness of an event that had never been utilised in this context before. Had we incorporated the added manipulation of an extended time period and discovered poor recall we would have been unable to establish whether this was due to factors associated with the target event, or factors associated with the interviewing process. Future research may seek to make comparisons between short and long passages of delay on recall of touch related information under the context of the current event.

The current study incorporates a relatively small sample of participants. As such, there were indications of effects that will require replication with an increased sample size to improve statistical power. Despite the small sample size, we were able to demonstrate important effects that warrant further investigation.

\section{General Discussion}

Overall we found that introducing a visual aid in the form of a HFD or a photograph of the child elicited a greater amount of touch-related information than verbal-only interviewing. This was not at the expensive of the accuracy of the information. Our finding is in contrast to previous studies that have reported increased 
errors in reporting with visual aid use (Brown et al., 2007; 2011; Steward et al., 1996; Willcock et al., 2006).

We found visual aids were most useful in assisting children to elaborate upon, or clarify instances of already reported touch. This reflects the findings of Teoh et al. (2010), particularly in reference to the age group of children that we included in our sample. These benefits were only apparent when visual aids were paired with open ended questioning. When visual aids were paired with more specific questions about touch, the accuracy of the information children reported dramatically declined. However, this was not limited to visual aid use and occurred across all interview conditions.

We found increased recall of touch information relative to previous studies (Bruck, 2009; Brown et al., 2007; 2011; Salmon et al., 2011; Willcock et al., 2006) in using our target event which was developed to incorporate instances of touch central to the nature of the event. In the context of an event where instances of touch appeared to be more salient and central to the event than has been previously studied, future research should seek to examine the effects of visual aids on recall in younger children. It may be that in younger children, photographs are more beneficial in supporting recall and reporting of touch information and thus may provide an effective visual aid to support verbal interviewing techniques.

In conclusion, at least with 9-11 year old children, visual aids in the form of a HFD or a photograph of the child, when used alongside best practise verbal interviewing techniques appear to facilitate children's detailed reporting of touch related information, without decreasing the accuracy of this information. It follows that they may be safe to use in the context of forensic interviewing under these conditions. 


\section{References}

Aldridge, J., \& Cameron, S. (1999). Interviewing child witnesses: Questioning techniques and the role of training. Applied Developmental Science, 3 (2), 136-147. doi:10.1207/s1532480xads0302_6

Aldridge, J., Lamb, M. E., Sternberg, K. J., Orbach, Y., Esplin, P. W., \& Bowler, L. (2004). Using a Human Figure Drawing to Elicit Information From Alleged Victims of Child Sexual Abuse. Journal of Consulting and Clinical Psychology, 72(2), 304-316. doi: 10.1037/0022-006X.72.2.304

Anderson, J., Martin, J., Mullen, P., Romans, S., \& Herbison, P. (1993). Prevalence of Childhood Sexual Abuse Experiences in a Community Sample of Women. Journal of the American Academy of Child \& Adolescent Psychiatry, 32(5), 911-919. doi: 10.1097/00004583-199309000-00004

Brown, D., \& Lamb, M. E. (2009). Forensic Interviews with Children: A Two-Way Street: Supporting Interviewers in Adhering to Best Practice Recommendations and Enhancing Children's Capabilities in Forensic Interviews. In K. a. C. Kuehnle, M. (Ed.), The Evaluation of Child Sexual Abuse Allegations: A Comprehensive Guide to Assessment and Testimony. (pp. 299-326). New Jersey: John Wiley \& Sons, Inc.

Brown, D. A., Pipe, M.-E., Lewis, C., Lamb, M. E., \& Orbach, Y. (2007). Supportive or Suggestive: Do Human Figure Drawings Help 5- to 7-Year-Old Children to Report Touch? Journal of Consulting and Clinical Psychology, 75(1), 33-42. doi: 10.1037/0022-006X.75.1.33

Brown D. A., Pipe, M.-E., Lewis, C., Lamb, M. E., \& Orbach, Y. (2011). How do body diagrams affect the accuracy and consistency of children's reports of 
bodily touch across repeated interviews? Applied Cognitive Psychology. doi: 10.1002/acp. 1828

Bruck, M. (2009). Human figure drawings and children's recall of touching. Journal of Experimental Psychology: Applied, 15(4), 361-374. doi: 10.1037/a0017120

Ceci, S.J., \& Bruck, M. (1993). Suggestibility of the child witness: A historical review and synthesis. Psychological Bulletin, 113 (3), 403-439. doi: 10.1037/00332909.113.3.403

DeLoache, J.S. (2000). Dual representation and young children's use of scale models/ Child Development, 71, 329-338. Retrieved from http://www.jstor.org/stable/1131993

DeLoache. J.S. (2004). Becoming symbol minded. Trends in Cognitive Sciences, 8, 66-70. doi:10.1016/j.tics.2003.12.004

DeLoache, J.S., \& Marzolf, D.P. (1992). When a picture is not worth a thousand words: Young children's understanding of pictures and models. Cognitive Development, 7, 312-329. doi:10.1016/0885-2014(92)90019-N

Deloache, J. S., \& Marzolf, D. P. (1995). The Use of Dolls to Interview Young Children: Issues of Symbolic Representation. Journal of Experimental Child Psychology, 60(1), 155-173. doi: 10.1006/jecp.1995.1036

Fanslow, J. L., Robinson, E. M., Crengle, S., \& Perese, L. (2007). Prevalence of child sexual abuse reported by a cross-sectional sample of New Zealand women. Child Abuse \& Neglect, 31(9), 935-945. doi: 10.1016/j.chiabu.2007.02.009

Field, A. (2009). Discovering Statistics using SPSS. London: SAGE Publications Ltd. Fleming, T.M., Merry, S.N., Robinson, E.M., Denny, S.J., \& Watson, P.D. (2007). Self reported suicide attempts and associated risk and protective factors 
amoung secondary school students in New Zealand. Australian and New Zealand Journal of Psychiatry, 41 (3), 213-221.

doi:10.1080/00048670601050481

Goodman, G.S., \& Reed, R.S. (1986). Age differences in eyewitness testimony. Law and Human Behaviour, 10 (4), 317- 332. Retrieved from http://www.jstor.org/stable/1393817

Klemfuss, J.Z., \& Ceci, S. (2009). Normative memory development and the child witness. In n Kuehnle, K. \& Connell. M. (Eds.), The Evaluation of Child Sexual Abuse Allegations: A Comprehensive Guide to Assessment and Testimony. (pp. 365-396). New Jersey: John Wiley \& Sons, Inc.

Lamb, M.E., Orbach, Y., Hershkowitz, I., Horowitz, D., \& Abbott, C.B. (2007). Does the type of prompt affect the accuracy of information provided by alleged victims of abuse in forensic interviews? Applied Cognitive Psychology, 21, 1117-1130. doi: 10.1002/acp.1318

Lamb, M.E., Orbach, Y., Hershkowitz, I., Esplin, P.W., \& Horowitz, D. (2007). A structured forensic interview protocol improves the quality and informativeness of investigative interviews with children: A review of research using the NICHD Investigative Interview Protocol. Child Abuse \& Neglect, 31, 1201-1231. doi:10.1016/j.chiabu.2007.03.021

Lamb, M.E., Orbach, Y., Sternberg, K.J., Aldridge, J., Pearson, S., Stewart, H.L., Esplin, P.W., \& Bowler, L. (2009). Use of a structured investigative protocol enhances the quality of investigative interviews will alleged victims of child sexual abuse in Britain. Applied Cognitive Psychology, 23, 449-467. doi: 10.1002/acp.1489 
Lamb, M.E., Sternberg, K.J., Orbach, Y., Esplin, P.W., Stewart, H., \& Mitchell, S. (2003). Age differences in young children's responses to open-ended invitations in the course of forensic interviews. Journal of Consulting and Clinical Psychology, 71 (5), 926-934. doi: 10:1037/0022-006X.71.5.926

Malloy, L.C., \& Quas, J.A. (2009). Children's suggestibility: Areas of consensus and controversy. In Kuehnle, K. \& Connell. M. (Eds.), The Evaluation of Child Sexual Abuse Allegations: A Comprehensive Guide to Assessment and Testimony. (pp. 365-396). New Jersey: John Wiley \& Sons, Inc.

Marin, B.V., Holmes, D.L., Guth, M., \& Kovac, P. (1979). The potential of children as eyewitnesses. Law and Human Behaviour, 3, 295-305. doi:

10.1007/BF01039808

Orbach, Y., Hershkowitz, I., Lamb, M.E., Sternberg, K.J., Esplin, P.W., \& Horowitz, D. (2000). Assessing the value of structured protocols for forensic interviews of alleged child abuse victims. Child Abuse \& Neglect, 24 (6), 733-752. doi:10.1016/S0145-2134(00)00137-X

Pipe, M.-E., Lamb, M. E., Orbach, Y., \& Esplin, P. W. (2004). Recent research on children's testimony about experienced and witnessed events. Developmental Review, 24(4), 440-468. doi: 10.1016/j.dr.2004.08.006

Pipe, M.-E., \& Salmon, K. (2009). Dolls, Drawing, Body Diagrams, and Other Props: Role of Props in Investigative Interviews. In Kuehnle, K. \& Connell. M. (Eds.), The Evaluation of Child Sexual Abuse Allegations: A Comprehensive Guide to Assessment and Testimony. (pp. 365-396). New Jersey: John Wiley \& Sons, Inc. 
Salmon, K. (2001). Remembering and reporting by children: The influence of cues and props. Clinical Psychology Review, 21(2), 267-300. doi: 10.1016/S02727358(99)00048-3

Salmon, K., Pipe, M.-E., Malloy, A., \& Mackay, K. (2011). Do non-verbal aids increase the effectiveness of 'best practice' verbal interview techniques? An experimental study. Applied Cognitive Psychology. doi: 10.1002/acp.1835

Steward, M. S., Steward, D. S., Farquhar, L., Myers, J. E. B., Reinhart, M., Welker, J. (1996). Interviewing Young Children about Body Touch and Handling. Monographs of the Society for Research in Child Development, 61(4/5), i-232. Retrieved from http://www.jstor.org/stable/1166205

Teoh, Y.-S., Yang, P.-J., Lamb, M. E., \& Larsson, A. S. (2010). Do human figure diagrams help alleged victims of sexual abuse provide elaborate and clear accounts of physical contact with alleged perpetrators? Applied Cognitive Psychology, 24(2), 287-300. doi: 10.1002/acp.1564

Willcock, E., Morgan, K., \& Hayne, H. (2006). Body maps do not facilitate children's reports of touch. Applied Cognitive Psychology, 20(5), 607-615. doi: 10.1002/acp.1212

Wilson, K. (2007). Forensic Interviewing in New Zealand. In M.-E. Pipe, Lamb, Michael, E., Orbach, Y., \& Cederborg, A-C. (Ed.), Child Sexual Abuse: Disclosure, Delay, and Denial (pp. 265-280). New Jersey: Lawrence Erlbaum Associates.

World Health Organiation (2010). Child Maltreatment. Retreived November 23, 2011, from http://www.who.int/mediacentre/factsheets/fs150/en/index.html 


\section{APPENDIX A}

\section{Parent information letter}

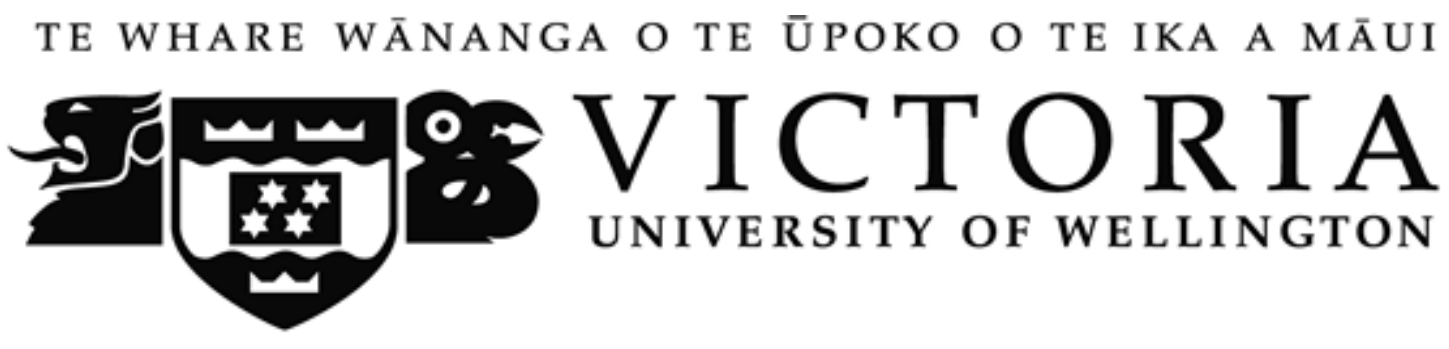

Using images to help children talk about their experiences

2011

Dear Parents/Caregivers,

We would like to invite your child to participate in a study about children's memory. We have the support of your child's school, and the study has been approved by the Victoria University School of Psychology Human Ethics Committee.

\section{What is the purpose of this research?}

We want to study what kinds of information children remember and describe about things they have experienced. We know that children's descriptions are usually made up of the actions that occurred ("what happened"), but are less likely to include the specific details about the event. One category of information that we are interested in is how well children remember bodily contact that has occurred during an interaction. Research of this kind can help develop guidelines for people talking with children in different contexts about their experiences (e.g., interviews about crimes they have witnessed or experienced, helping doctors talk to children about their symptoms). Who is conducting the research?

- This study is being conducted by Dr Deirdre Brown and Wendy Higgs (Master's student) from the School of Psychology at Victoria University, Wellington; Professor Michael Lamb from Cambridge University, UK; and Professor Margaret-Ellen Pipe from the City University of New York, USA.

What is involved if your child participates in this study?

- Your child's class will take part in an interactive lesson about health and safety. Children will work in small groups, with an adult group leader. The event will include some instances of body contact between your child and their partner, and between them and the researcher. For example, children will have their ears touched whilst having their temperature measured with a digital thermometer. In another activity children will listen to each other's heartbeats using a stethoscope (and thereby touching their partner's back and chest), and practice finding a pulse on their partner (touching their partner's neck and wrist).

- Between 1 and 2 weeks after the event your child will be interviewed, individually, at school, to see what they can remember and describe about the event. During the interview we will ask your child different types of questions;

○ We will ask them to tell us everything they can about what happened and then use broad (e.g., tell me more) and specific questions to help them tell any more details they remember 
- We will ask some questions about things that did not happen during the event e.g. "did your leader put the thermometer under your foot?"

- Some of the children will be asked to indicate on either a drawing of a child or a photo that was taken at the beginning of the interview where any body contact occurred during the activities e.g., where their partner put a plaster on them

- Your child can indicate that he or she does not wish to proceed at any stage of the study and will be excused.

- Children who are interviewed will receive a small gift (e.g., stickers) to thank them. Book vouchers will also be donated to the school library.

\section{Privacy and Confidentiality}

- Consent forms, photographs, line drawings and video recordings will be kept for five years after publication and then destroyed.

- The data will be coded by numbers and therefore your child will never be identified individually. The dataset (the numbers) will be kept indefinitely and will be securely stored in the laboratory of Dr. Deirdre Brown.

- Coded data (that is, without your child's name) may be shared with other competent professionals upon request, and may also be used in other studies.

\section{What happens to the information that you and your child provide?}

- We may publish the results of the study in a scientific journal or present them in a conference. No child will be identified in the results.

- You may wish to give your permission for parts of your child's interview to be included in conference presentations. If you do not wish to give consent for this you may still consent to your child participating in the study.

- Only researchers associated with the project will have access to the information reported by your child, although, in the unlikely event that your child reports witnessing or being a victim of a crime, we would also inform the authorities.

The results of the study will be available in approximately March 2012. A summary of our major findings will be sent out to you at the end of the study.

If you have any further questions regarding this study, you are most welcome to contact Dr. Deirdre Brown, ph 4635233 ext 8059 or Deirdre.Brown@vuw.ac.nz

If you agree for your child to participate in this study, please return this consent form to your child's teacher by Date. Please also return the form if you do not wish your child to be interviewed about the class event.

If you allow your child to participate in the study please do not talk about the class event, or the interview with your child until after the study is finished.

Thank you for your time in considering participating in this study.

Yours sincerely,

Deirdre Brown, PhD, PgDipClPs, MNZCCP

Lecturer in Clinical and Forensic Psychology

School of Psychology

Victoria University of Wellington 


\section{APPENDIX B}

\section{Consent form}

TE WHARE WĀNANGA O TE ŪPOKO O TE IKA A MĀUI

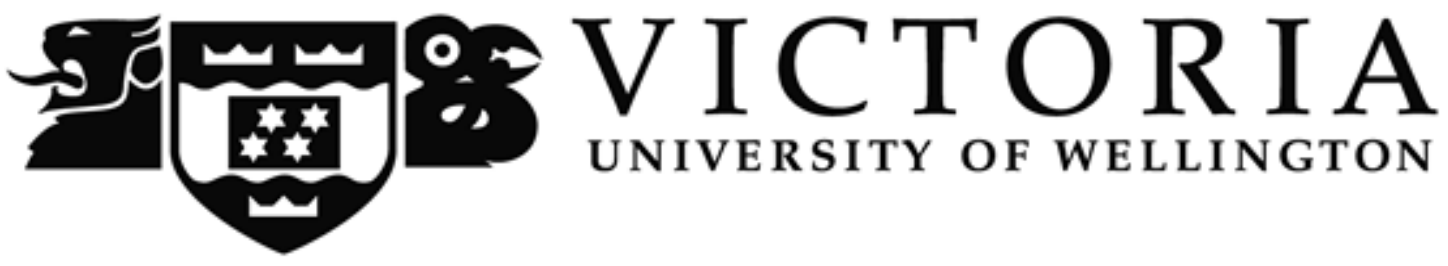

\section{Using images to help children talk about their experiences}

\section{Statement of Consent}

I have read all the information above and have asked any questions relating to this study, which have been answered satisfactorily.

\section{Please tick the statement that applies:}

I consent to my child participating in this research. My child can indicate they do not wish to proceed at any stage of the study, and they will be excused.

I do not wish my child to be interviewed about the class event.

I do not wish my child to take part in the class event.

Child's Name: Date of Birth:

Parent's Name:

Email Address (optional):

Signature:

Date:

\section{Consent for use of photographs and/or video excerpts}

Please tick any that apply (if you do not select one of these options your child's video and photograph will not be used in any presentations or publications)

I give permission for parts of the video of my child's interview to be used in conference presentations. I understand that my child will not be referred to by name nor any identifying information provided

I give permission for my child's photograph, used in the interview about the health event, to be included in conference presentations or publications. I understand that my child will not be referred to by name nor any identifying information provided 
APPENDIX C

Human Figure Diagram

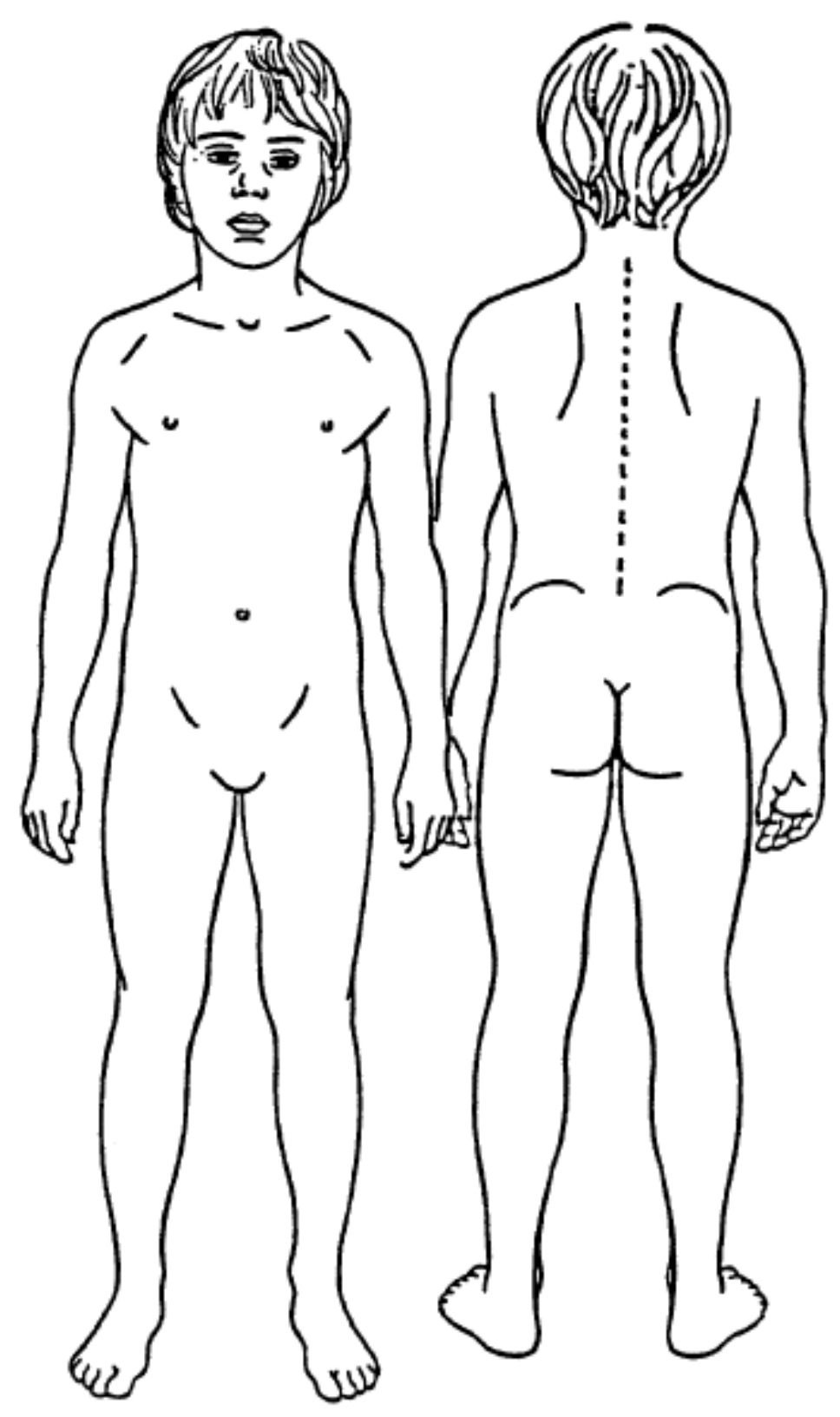




\section{APPENDIX D}

\section{Interview script}

Record the following information on the tape before you begin

My name is . The date is . I am interviewing

(code number), at (time).

Section I. TRUTH/LIES CEREMONY AND RULES OF INTERVIEW

Well, , my name is . My job is to talk to children about things that have happened to them. So, before we begin, I want to make sure that you know the difference between things that are true and not true. If I said that you took your shoes off when you came into this room, would that be true or not true?

[Wait for an answer. If the answer was incorrect say:]

Let's look at your feet. Have you got your shoes on? [Wait for an answer ("Yes")]

So, that would not be true, because you didn't take your shoes off.

[If the answer was correct say:] Great, I see that you know what telling the truth means.

When we talk today you should only tell me about things that are really true, that really happened to you.

If you don't understand something, you can just say "I don't understand".

[Pause]

And if you don't know the answer to something, just say, "I don't know". Let's practice that. If I ask you what's in my pocket what would you say?

[Wait for an answer, then say:]

Right, you don't know, do you?

[If child offers a guess, say:]

No, you don't know what's in my pocket because you haven't seen in there.

When you don't know, you don't have to guess, it's okay to say "I don't know".

[Pause]

And if I say things that are wrong, you should correct me. Okay? 
[Pause]

So, if I said that you were a girl/boy (whatever they aren't) what would you say?

[Wait for an answer. If the answer was incorrect or no response say:]

And are you a boy/girl? [Wait for an answer]

That's right. Now you know you should correct me if I make a mistake or say something wrong.

So let's practice that one more time. If I said that you were standing up, what would you say?

[Wait for an answer] That's great. So while we are talking today it's okay for you to tell me if I make a mistake or say something that is not true, and remember you don't have to guess, it's okay to say when you don't know something.

Section II. RAPPORT (PRACTICE IN EPISODIC MEMORY)

Now, I want to get to know you better.

Tell me about all the things that you've done today, from the time you woke up/lunch time until the time you came here and met me.

[Wait for child's answer]

Tell me more about [what you did this morning]

[Wait for child's answer] [Note: use this prompt as often as needed throughout this section]

\section{Then what happened?}

[Wait for child's answer] [Note: use this prompt as often as needed throughout this section]

Tell me everything that happened after [some activity or portion of the event mentioned by the child] until you came here.

[Wait for child's answer]

What was the very next thing that happened after [some activity or portion of the event mentioned by the child]?

[Wait for child's answer]

You told me you [activity mentioned by child]. Tell me everything about [activity mentioned by child]. 
[Wait for child's answer] [Note: use this prompt as often as needed throughout this section]

\section{Section III. INTERVIEW ABOUT STAGED EVENT}

Now that I know you a little better, let me tell you why I've come to talk to you today.

1. I heard that a couple of weeks ago some people came to talk to your class about health and safety. I wasn't there but I'd like to know all about what happened. Tell me everything that happened from the beginning to the end.

[Wait for the child to answer. If child gives a brief description, proceed to follow-up questions. If the child does not provide any information about the event, wait then say:]

2. I heard that you and your class went into the performance room and learned about health and safety. Tell me all about what happened.

[Wait. If child begins to talk about event, proceed to follow-up questions. If child does not discuss the event, proceed to Question 3]

3. I heard that after they talked about safety they gave you some stickers. Tell me all about what happened.

[Wait for a response. If child does not provide any information proceed to Question 4. If child begins to talk about event, proceed to follow-up questions]

4. I heard you learned about hearts, and listened to them. Tell me all about what happened.

\section{Section IV. FOLLOW-UP QUESTIONS}

Follow up each piece of information reported by child (e.g., activities, people, location, items present) and encourage elaborative reporting. Try to be systematic with this, focusing on one event and details associated with it until child indicates s/he can recall no more, before moving to next activity/piece of information. Use the following prompts:

- REPEAT WHAT CHILD HAS SAID, USING HIS/HER WORDS [remember not to provide details, (including names) that the child hasn't mentioned] (e.g., OK, so you had to listen to someone's breathing) then say: Tell me everything you remember about that.) 
- And then what happened? [You can use this prompt several times until you have an overview of the incident]

- Think back to that time and tell me everything that happened from [some preceding event mentioned by the child] until [event as described by child]

- Tell me more about [something or event mentioned by child] [You can use this prompt many times]

- Tell me some more things about [something or event mentioned by child] [You can use this prompt many times]

BREAK: Check with the monitor once you have exhausted your line of questioning, or feel that the child needs a break. Take as many as you need to ensure you are asking best questions possible and maintaining child's motivation/interest. While you take a break, ask the child to think really hard about everything that happened that day, and that you will ask if they remember anything else when you return.

NOTE: AFTER TAKING A BREAK TO REVIEW THE CHILD'S COMMENTS, YOU MAY WANT TO ASK ADDITIONAL PAIRED CUE (SECTION IV) AND PAIRED LEADING (SECTION V) QUESTIONS.

\section{Section V. PROBES ABOUT SPECIFIC INFORMATION FOCUSING ON DETAILS}

1a. You said something about [something the child said, e.g., getting a sticking plaster on] Tell me everything about that.

[Wait for a response]

1b. You said something about [something the child said, e.g., listening to the heart] Tell me everything about that.

[Wait for a response]

1c. You said something about [something the child said, e.g., getting how tall you are done] Tell me everything about that.

[Wait for a response]

Use as many of these as you need. 
2. If the central information remains sparse (e.g., we did some stuff about being safe) ask I don't understand what the safe things were that you did. Tell me everything about that so that I can understand.

[Use as many such prompts as you need. Follow up with cue questions like 1a, 1b, and $1 \mathrm{c}$ in this section to probe further]

\section{Section VI. PROBES ABOUT INFORMATION NOT MENTIONED BY THE} CHILD

Things we want to have heard about by the end:

- Activity
○ Hearts
○ Temperatures
- Hazards
- Cuts

- Groups

○ Leader of each activity

- Partner for temperature

○ Partner for hearts

- Location

- First activity

[Leading non-suggestive questions should only be asked after you have tried nonleading approaches and feel you are missing forensically crucial information. If such information is missing (e.g., what happened during particular activities, who they had as partners for events) ask leading questions such as:]

\section{Who was your partner?}

[Wait for a response.] Tell me all about that.

\section{Who's heart did you listen to?}

[Wait for a response and follow affirmative responses with:] Tell me what happened.

3. If child has not identified their group leader, ask Tell me who your group leader was.

[Wait for a response. If child provides information, follow-up with:] Tell me more things about her. 
[Follow up with further directed questions as necessary, e.g., Tell me about what she looked liked].

4. If child has not identified place of event, ask Where did you go to learn about safety?

\section{[SIGN POST HERE THAT YOU ARE STARTING SOMETHING NEW]. I}

want to be really sure I understand everything you told me, so I need to ask you some more questions. I might ask you again about things you've already talked about, but that's ok, just tell me again. Remember I only want you to tell me things that you know really happened, you don't have to guess, and it's ok to say "I don't know" if you're not sure about something.

\section{THEN EITHER:}

a. Remember when you first came in I took your picture? Well here is the picture of you. I want to make sure I remember everything you told me today. Show me on the picture exactly where [repeat any statements about touch during verbal interview, e.g., Jane put the listening on you, Jane put the stethoscope on your back]. Give the child a dot sticker each time they talk about a new body place and get them to put it on the picture where they were touched. [make sure they use the front and the back of the picture, point out front and back if necessary]. So you told me about that before, but is there anything else you can tell me about that?

b. See this picture? This picture is a child just like you. I want to make sure I remember everything you told me today. Show me on the picture 
exactly where [repeat any statements about touch during verbal interview, e.g., Jane put the listening on you, Jane put the stethoscope on your back]. Give the child a dot sticker each time they talk about a new body place and get them to put it on the picture where they were touched. [make sure they use the front and the back of the picture, point out front and back if necessary]. So you told me about that before, but is there anything else you can tell me about that?

c. I want to make sure I remember everything you told me today. You told me that Jane put the listening on you, Jane put the stethoscope on your back - can you tell me again exactly where on your body that was? So you told me about that before, but is there anything else you can tell me about that?

Great. You've shown/told me lots of different places on your body that things happened while you were learning about health and safety.

If the child has provided any information about touching, follow up with: Did anything happen on any other part of your body? [If child responds affirmatively say: Tell me everything about that. Follow up with other open-ended prompts [such as Tell me more about that or And then what happened?] until the child provides no new information. Be sure that the elicited information is not ambiguous, use focused questions to clarify if necessary. Get child to show and mark on the photo where the touch occurred. 
Once the child has marked on the photo/body map all of the touch that was reported in the verbal interview, proceed to the specific questions. Only ask questions if they $\underline{\text { have not already been reported by child in their free recall reports. }}$

\section{Specific touch questions:}

If the child denies touching to the first question, ask the second question which provides a label for the action associated with the touching. If they describe an incident of touch in response to the first question, move to the next body area. All underlined questions should be asked.

\section{Did [child's partner] touch you on your chest?}

[Wait for a response]

[If child responds affirmatively, say:] Tell me everything about that.

- Did [child's partner] put the stethoscope on your chest?

[Wait for a response]

[If child responds affirmatively, say:] Tell me everything about that.

2. Did [child's partner] touch you on your wrist?

[Wait for a response]

[If child responds affirmatively, say:] Tell me everything about that.

- Did [child's partner] put their fingers on your wrist?

[Wait for a response]

[If child responds affirmatively, say:] Tell me everything about that.

3. Did [child's partner] touch you on your forehead?

[Wait for a response]

[If child responds affirmatively, say:] Tell me everything about that.

- Did [child's partner] put their hand on your forehead? 
[Wait for a response]

[If child responds affirmatively, say:] Tell me everything about that.

4. Did [not child's partner] touch you on your ear?

[Wait for a response]

[If child responds affirmatively, say:] Tell me everything about that.

- Did [not child's partner] put a thermometer in your ear?

[Wait for a response]

[If child responds affirmatively, say:] Tell me everything about that.

\section{Did [child's partner] touch you on your arm?}

[Wait for a response]

[If child responds affirmatively, say:] Tell me everything about that.

- Did [child's partner] put the stethoscope on your arm?

[Wait for a response]

[If child responds affirmatively, say:] Tell me everything about that.

6. Did[child's partner] touch you on the elbow?

[Wait for a response]

[If child responds affirmatively, say:] Tell me everything about that.

- Did [child's partner] put their fingers on your elbow?

[Wait for a response]

[If child responds affirmatively, say:] Tell me everything about that.

7. Did [child's partner] touch you on your tummy?

[Wait for a response]

[If child responds affirmatively, say:] Tell me everything about that.

- Did [child's partner] put the stethoscope on your tummy?

[Wait for a response] 
[If child responds affirmatively, say:] Tell me everything about that.

8. Did [child's partner] touch you on the mouth?

[Wait for a response]

[If child responds affirmatively, say:] Tell me everything about that.

- Did [child's partner] put the thermometer in your mouth?

[Wait for a response]

[If child responds affirmatively, say:] Tell me everything about that.

9. Did [not child's partner] touch you on the back?

[Wait for a response]

[If child responds affirmatively, say:] Tell me everything about that.

- Did [not child's partner] put the stethoscope on your back?

[Wait for a response]

[If child responds affirmatively, say:] Tell me everything about that.

10. Did [child's partner] touch you on your neck?

[Wait for a response]

[If child responds affirmatively, say:] Tell me everything about that.

- Did [child's partner] put their fingers on your neck?

[Wait for a response]

[If child responds affirmatively, say:] Tell me everything about that.

11. Did [child's partner] touch you on the back?

[Wait for a response]

[If child responds affirmatively, say:] Tell me everything about that.

- Did [child's partner] put the stethoscope on your back?

[Wait for a response]

[If child responds affirmatively, say:] Tell me everything about that. 
12. Did [child's partner] touch you on the knee?

[Wait for a response]

[If child responds affirmatively, say:] Tell me everything about that.

- Did [child's partner] put the thermometer under your knee?

[Wait for a response]

[If child responds affirmatively, say:] Tell me everything about that.

13. Did [child's partner] touch you on your ankle?

[Wait for a response]

[If child responds affirmatively, say:] Tell me everything about that.

- Did [child's partner] put their fingers on your ankle?

[Wait for a response]

[If child responds affirmatively, say:] Tell me everything about that.

14. Did [child's partner] touch you under your arm?

[Wait for a response]

[If child responds affirmatively, say:] Tell me everything about that.

- Did [child's partner] put a thermometer under your arm?

[Wait for a response]

[If child responds affirmatively, say:] Tell me everything about that.

\section{Did [not child's partner] touch you on your wrist?}

[Wait for a response]

[If child responds affirmatively, say:] Tell me everything about that.

- Did [not child's partner] put her/his fingers on your wrist?

[Wait for a response]

[If child responds affirmatively, say:] Tell me everything about that. 
16. Did [child's partner] touch you on your ear?

[Wait for a response]

[If child responds affirmatively, say:] Tell me everything about that.

- Did [child's partner] put a thermometer in your ear?

[Wait for a response]

[If child responds affirmatively, say:] Tell me everything about that. 


\section{APPENDIX E}

Hearts

Partner's name

Some mention/description of stethoscope

Listening to hearts

Chest

Back

Stomach

Taking pulses

Wrist

Ankle

Neck

Group leader

$\underline{\text { Temperatures }}$

$\square$ Some mention/description of thermometer

2 different kinds of thermometer

Partner's name

Taking temperatures

Temperature chart, writing on it

Ear

Behind knee

Under arm

Wipes

Group leader

Forehead

$\underline{\text { Cuts }}$

Arthur video clip, power point

Drawing cut with red pen

Putting on pressure

Raising above head

Wiping with antibacterial wipe

Putting on plaster

Group leader $\underline{\text { Hazards }}$

Group leader

Identifying hazards

Why they're unsafe

How to improve

Some description of some

$\underline{\text { General }}$

Coming from class

Getting into groups

Who was in their group

4 stations

Getting stickers at the end

Different leaders at each 


\section{APPENDIX F}

\section{Coding Manuel}

Recommended coding process:

1 The transcript will be read through marking where each section of the interview (verbal, touch follow up and specific touch questions) begins, and any touch related information will be highlighted.

2 The touch table will be filled in, marking whether or not each touch was reported and whether it was correct, an incorrect intrusion or an incorrect distortion. If there were any incorrectly reported touches they will be noted underneath the table.

3 The highlighted touch information in the transcript will be read through and pieces of information will be identified and circled.

4 Each piece of information will be considered and coded according to its accuracy as correct, incorrect distortion, incorrect intrusion, repeated, other, prompted correct, prompted incorrect distortion, or prompted incorrect intrusion. The letter representing its accuracy will be written above the piece of information.

5 The coding table tallying pieces information according to their accuracy will be filled out.

6 The touch specific questions table noting whether questions were asked or not, whether 1 part of the question or 2 parts of the question were asked, and whether the response was correct, an incorrect distortion, an incorrect intrusion, not reported, or incorrectly asked by the interviewer will be filled out. Any incorrect responses will be described underneath the table. 
Phase of Interview: Data will be coded according to the phase of the interview it was elicited in.

Free recall: This phase of the interview runs from the end of the free narrative practise until the time that the touch specific follow up begins. The only information to be coded in this section is that related to touch including; where it occurred eg. "we measured the heartbeat on the chest", who was involved "my partner measured my heartbeat", what was involved "we measured temperatures with a thermometer". The beginning of this phase will be indicated by the interviewer stating;

"I heard that a couple of weeks ago some people came to talk to your class about health and safety, I wasn't there but I'd like to know everything that happened, so tell me everything that happened from the beginning to the end" Touch follow-up: This may be indicated in the script by the interviewer stating;

"we're going to do something a bit different now"

By the interviewer introducing a visual aid

"See this picture, this is a child just like you"

"Remember when you first came in and I took your picture? Here's your picture"

By indicating they will be asking further questions

“I want to remember everything you've told me today, so I need to ask you some more questions"

Touch specific questions: This begins when the interviewer begins asking specific "did your partner" questions.

\section{Checklist of touches}

Using the provided table (depicted below) each scripted touch will be ticked off in the 'yes' column if it is reported. If it is reported for the first time in the verbal section of 
the interview the letter $\mathrm{V}$ will be written into the 'phase' column. If the touch is reported for the first time in the follow up touch section of the interview the letter $\mathrm{F}$ will be written into the 'phase' column. If the touch is reported for the first time in the specific touch questions section of the interview the letter $\mathrm{S}$ will be written into the 'phase' column. If the reported touch is correct a tick will be placed in the 'correct' column. If the touch was incorrect and the reported information is a distortion of the correct touch, a tick will be place in the 'incorrect - distortion column'. A distortion describes a scripted touch that is reported wrongly - for example the right touch but the wrong person or agent. If a touch is reported which is not scripted at all a tick will be placed in the 'incorrect- intrusion column'. If a scripted touch is not reported in any phase of the interview, a tick will be placed in the 'no' column. A description of all incorrect touches reported should be noted underneath the table.

Subject number:

\begin{tabular}{|l|l|l|l|l|l|l|}
\hline & Scripted & Reported & & \multicolumn{2}{l|}{ Incorrect } \\
\hline Phase & Touch & Yes & No & Correct & Distortion & Intrusion \\
\hline & Chest & & & & & \\
\hline & Back & & & & & \\
\hline & Stomach & & & & & \\
\hline & Wrist & & & & & \\
\hline & Neck & & & & & \\
\hline & Ankle & & & & & \\
\hline & Forehead & & & & & \\
\hline & Ear & & & & & \\
\hline & Armpit & & & & & \\
\hline & Knee & & & & & \\
\hline & Fake & & & & & \\
\hline & Arm & & & & & \\
\hline & Mouth & & & & & \\
\hline & Elbow & & & & & \\
\hline
\end{tabular}

Phase key: V (verbal), F (touch follow up), S (specific touch questions) If an incorrect touch was reported please document what the incorrectly reported information was below: 


\section{$\underline{\text { Touch specific questions checklist }}$}

Using the table provided (depicted below) each question asked during the touch specific section of the interview will be recorded. If a question was asked a $\mathrm{Y}$ for yes will be placed in the Asked $\mathrm{Y} / \mathrm{N} \& 1 / 2$ column, if the question was not asked a $\mathrm{N}$ for no will be placed in the $\mathrm{Y} / \mathrm{N} \& 1 / 2$ column. If the first part of the question was asked a 1 will be placed in the $\mathrm{Y} / \mathrm{N} \& 1 / 2$ column. If both parts of the question were asked $1+2$ will be placed in the $\mathrm{Y} / \mathrm{N} \& 1 / 2$ column. Eg. Part one of a question: "Did (name) touch you on the knee?" Eg. Part two of a question: “Did (name) put a thermometer under your knee?"

In the case that an interviewer mistakenly asks a question that should not have been asked, the information the child provides in response will be disregarded and not coded. Questions that should not be asked include; asking about a touch that was scripted but has already been reported in previous parts of the interview, or, asking the second part of a question when the answer to the first part of the question was affirmative, eg. Interviewer: 'Did (name) touch you on the forehead?' Child: 'Yes' Interviewer: 'Did (name put their hand on your forehead?'. 'Incorrectly asked' will also be written in the Asked $\mathrm{Y} / \mathrm{N} \& 1 / 2$ column if a question is asked that should not have been.

If a question is asked about a scripted touch and it produces a 'no' response, 'not reported' will be written in the Asked Y/N \& 1/2 column. 
Touch Specific Questions

\begin{tabular}{|l|l|l|l|l|}
\hline Question & $\begin{array}{l}\text { Asked Y/N \& } \\
\mathbf{1 / 2}\end{array}$ & Correct & Incorrect -D & Incorrect - I \\
\hline Ear & & & & \\
\hline Forehead & & & & \\
\hline Neck & & & & \\
\hline Armpit & & & & \\
\hline Knee & & & & \\
\hline Chest & & & & \\
\hline Back & & & & \\
\hline Stomach & & & & \\
\hline Ankle & & & & \\
\hline Wrist & & & & \\
\hline Fake-arm & & & & \\
\hline Fake-mouth & & & & \\
\hline Fake-elbow & & & & \\
\hline Fake person ear & & & & \\
\hline $\begin{array}{l}\text { Fake person } \\
\text { back }\end{array}$ & & & & \\
\hline $\begin{array}{l}\text { Fake person } \\
\text { wrist }\end{array}$ & & & & \\
\hline Total & & & & \\
\hline
\end{tabular}

Key: Under asked $\mathrm{Y}$ for yes, $\mathrm{N}$ for no, 1 for first part of question asked, 2 for second part of question asked. $\mathrm{D}=$ distortion, $\mathrm{I}=$ intrusion. If the question was a scripted touch and the answer was no, write 'not reported' in the Asked Y/N \& 1/2 column. If as question was incorrectly asked by the interviewer (see coding notes) write 'incorrectly asked' in the Asked Y/N \& 1/2 column.

\section{Detail}

Pieces of information: Touch information will be coded according to meaningful chunks, each utterance that adds a new detail in the description of a touch will be counted as a 'piece of information' and tallied. Information will be coded only once. For example: Phrase "So I was with R that time. And we had to check for a heartbeat on our heart, on our wrist I think, and on the back"

Chunked into "I was with R that time" (who), "we had to check for a heartbeat" (what), "on our heart" (where), "on our wrist" (where), "and on the back" (where). Only information directly relevant to touch will be coded. Information reported at the same station but not relevant to touch will not be coded. E.g. "we wrote our knee temperature on the sheet". 
Correct $(C)$ : This is used to code pieces of information that accurately reflect what did happen during the health and safety event.

Incorrect-Distortion (I-D): This is used to code pieces of information that did happen but not in the way that the child reports e.g. attributing an action that did occur to the wrong person or agent. E.g. "we put the stethoscope on our neck".

Incorrect-Intrusion (I-I): This is used to code reported pieces of information that did not occur at all. Eg. "we put the thermometer in our mouth to measure the temperature".

Prompted $(P)$ : This is used to code information that is reported in response to a direct question from the interviewer when the child has not previously provided the information. This applies mostly to the touch-specific question phase of the interview eg. Interviewer asks "Did (name) touch you on the tummy?" Child responds "yes". Prompted information will be coded as prompted - correct $(P-C)$, promptedincorrect distortion (P-ID), or prompted incorrect intrusion $(P-I I)$ depending upon accuracy. Any information provided spontaneously after the initial yes is coded as normal and not coded as prompted. E.g. Interviewer: "did (name) touch you on the knee?" Child: “yes", Interviewer: "tell me everything about that", Child: "He put the thermometer under my knee to see how hot it was". In this case, "yes" would be coded as prompted-correct, but "he put the thermometer under" and "to see how hot it was" would be coded as correct, and not prompted. 
In the case that an interviewer mistakenly brings in new information during the first 2 sections of the interview, the verbal section or the touch follow up section, the child's response will also be coded as prompted - correct, incorrect distortion or incorrect intrusion. Any information provided spontaneously after the initial response to the prompt will not be coded as prompted.

Other $(O)$ : This is used to code ambiguous, unverifiable or subjective pieces of touch information. Ambiguous pieces of information related to touch include statements that lack sufficient clarity to determine what is referred to and where elaboration does not occur e.g. "I did it to him and he did it to me". Unverifiable pieces of information include statements where is it impossible to determine accuracy, i.e. anything nonscript related that was not recorded on video or in note form e.g. "I listened to his heart but I couldn't hear it". Subjective pieces of information include statements that reflect opinion e.g. "the stethoscope was cold". Ensure to only code touch related information.

Don't Know (DK): This is used to code 'don't know', 'I don't remember', or any equivalent answers in response to specific touch questions during the last phase of the interviewing,

Repeated $(R)$ : This is used to code pieces of information that have been identified as touch information in the transcript but are a repeat of previously coded information. Repeated information is used for coding reliability only; it will not be included in analysis and thus is not included in the table tallying pieces of information and accuracy. 
Using the table provided (depicted below) the pieces of information elicited in each phase of the interview will be tallied according to their accuracy.

Child Info

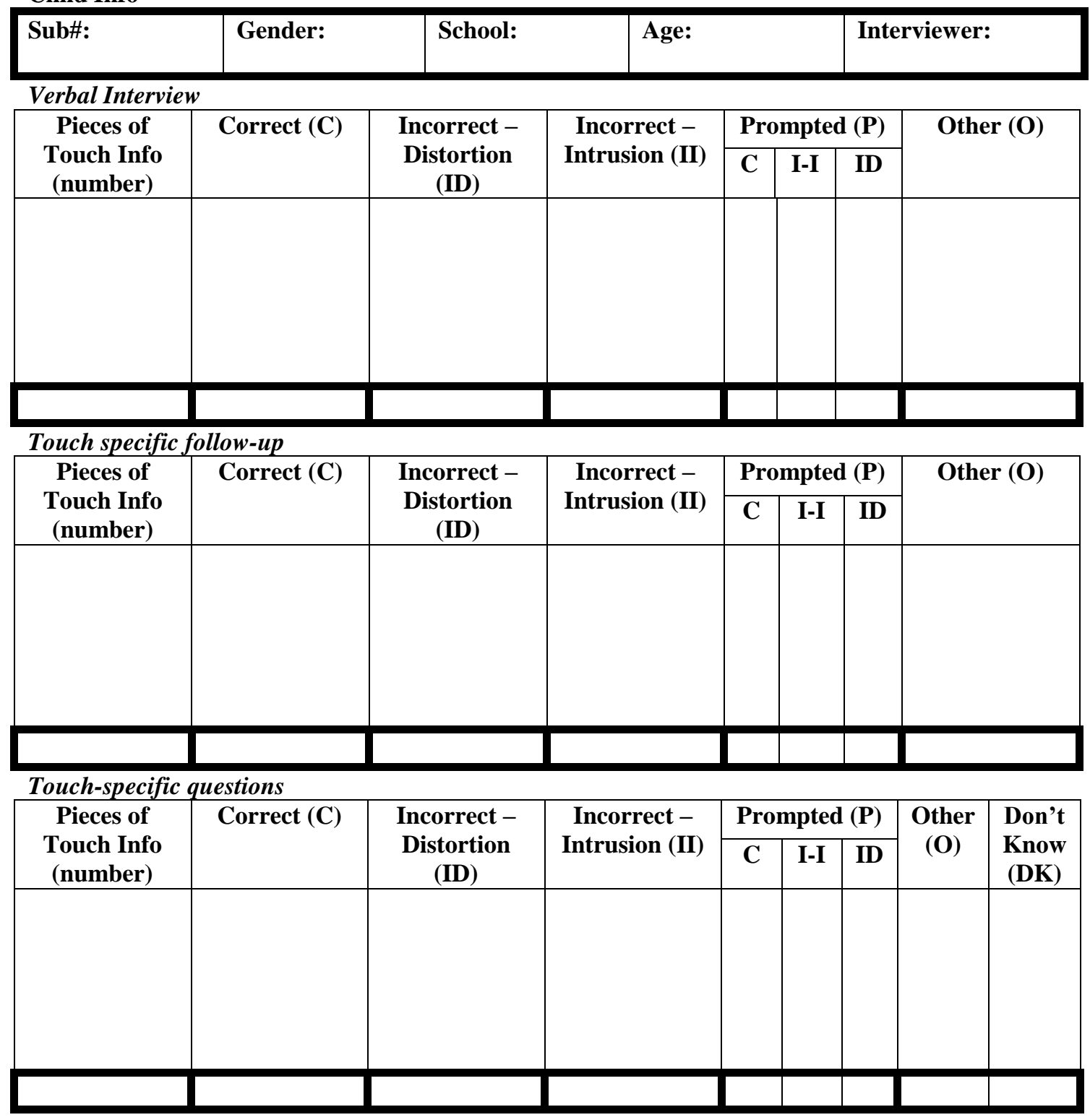

\section{Examples of correct statements}

\section{$\underline{\text { Touch components }}$}

Relevant touch related information includes:

\section{Who was involved}

- That they had a partner 
- Who the partner was

- That there was a group leader

"I went with the person next to me"

"I think it was (name of partner)"

"we had to have a partner"

"we got into pairs"

"someone else did it to us"

\section{Where it happened}

- Don't worry about left or right

- Don't worry about top or bottom of wrist

"under their arm"

"under their knee"

"in their ear"

"we felt it on their front"

\section{What happened}

- Naming the activity gets included as correct once

"we went to thermometers"

"we had to put a thermometer under their arm"

"we had to do the temperature"

"we had to feel our partner's heartbeat"

"we felt each other's heart beat"

\section{What they were touched with}

"the one with the stethoscope... we put it over by their heart"

"we got a stethoscope...we had to feel their heartbeat" 
"we put the thermometer under our armpit"

"he put the back of his hand on my head"

"he just got his two fingers and put them on my wrist"

\section{Why it happened}

"to see if we could hear our heart beat"

" to see how hot it was"

"to try to feel the pulse"

\section{$\underline{\text { Temperature or thermometer activity components }}$}

- Measured temperature (this implies some kind of bodily contact occurred)

- Measured temperature with a partner

- Partner's had turns

- What they specifically did with their partner

- Measured temperature in the ear

"when you measured your partner's ear"

"we used the thermometer in the ear"

- Measured temperature under the arm

- Measured temperature under the knee

"under their leg" this is fine - it doesn't have to be under the knee, pointing is also fine

- Felt the forehead

- Used a thermometer to measure the temperature

- The process of using the thermometer related to physical contact

"I did (child's name's), put it in, it went beep, took it out"

- Used two different kinds of thermometers to measure temperature 
- Mention of a group leader as part of the activity

\section{What not to include}

- Any descriptions of thermometers

- Any descriptions of the temperature recording sheet or writing on the recording sheet, or mention of body parts in relation to the temperature recording sheet.

- Any specific measurements

\section{Heartbeat or stethoscope activity components}

- Listened for heartbeat (this implies some kind of bodily contact occurred)

"we had to try and find the persons heartbeat"

"we had to feel our partner's heartbeat"

- Measured heartbeat/pulse with a partner

"also we did pulses with our partners"

- Who their partner was

"I had (child's name) for that one"

- Specifically what they did with their partner

- That they took turns

- Listened for the heartbeat on the chest - on the heart is fine don't have to say chest

"I had the stethoscope, and I put it on R's chest"

"[points to chest]"

"we had to feel their heartbeat... we felt it on their front"

- Listened for the heartbeat on the back 
"we had to put the stethoscope on their back"

- Listened for the heartbeat on the stomach

- Felt for pulse

"we had to feel the pulse"

- Felt for the pulse on the wrist

"we used our two fingers to... to put them on the wrist"

"we had to feel it on our wrist"

- Felt for the pulse on the neck

"we had to put our two fingers on our partners neck"

- Felt for the pulse on the ankle

- Used a stethoscope to hear/measure/listen to the heartbeat

- Used their fingers to feel for the pulse

- The process of measuring the heartbeat or pulse

- Mention of a group leader as part of the activity

\section{What not to include}

- Whether or not the child actually was able to hear the heartbeat or feel the pulse

- Any description of what the heart beat/pulse was doing

\section{Other}

- In the case that a child reports something that is incorrect and then promptly corrects themselves, disregard the incorrect information.

- If I child reports something but then retracts it later in the interview, code each piece of information separately. 
- If I child reports doing activities to themselves, consider it an incorrect distortion.

- If I child reports something but says they're not sure, go ahead and code the information.

- If a child reports something correctly and then adds an incorrect detail later, code each piece of information separately. 